\title{
How self-organized criticality works: A unified mean-field picture
}

\author{
Alessandro Vespignani \\ International Centre for Theoretical Physics (ICTP), P.O. Box 586, 34100 Trieste, Italy
}

\author{
Stefano Zapperi \\ Center for Polymer Studies and Department of Physics, Boston University, Boston, Massachusetts 02215
}

(Received 17 September 1997; revised manuscript received 17 December 1997)

\begin{abstract}
We present a unified dynamical mean-field theory, based on the single site approximation to the masterequation, for stochastic self-organized critical models. In particular, we analyze in detail the properties of sandpile and forest-fire (FF) models. In analogy with other nonequilibrium critical phenomena, we identify an order parameter with the density of "active"' sites, and control parameters with the driving rates. Depending on the values of the control parameters, the system is shown to reach a subcritical (absorbing) or supercritical (active) stationary state. Criticality is analyzed in terms of the singularities of the zero-field susceptibility. In the limit of vanishing control parameters, the stationary state displays scaling characteristics of self-organized criticality (SOC). We show that this limit corresponds to the breakdown of space-time locality in the dynamical rules of the models. We define a complete set of critical exponents, describing the scaling of order parameter, response functions, susceptibility and correlation length in the subcritical and supercritical states. In the subcritical state, the response of the system to small perturbations takes place in avalanches. We analyze their scaling behavior in relation with branching processes. In sandpile models, because of conservation laws, a critical exponents subset displays mean-field values $\left(\nu=\frac{1}{2}\right.$ and $\left.\gamma=1\right)$ in any dimensions. We treat bulk and boundary dissipation and introduce a critical exponent relating dissipation and finite size effects. We present numerical simulations that confirm our results. In the case of the forest-fire model, our approach can distinguish between different regimes (SOC-FF and deterministic FF) studied in the literature, and determine the full spectrum of critical exponents. [S1063-651X(98)09805-5]
\end{abstract}

PACS number(s): 64.60.Lx, 05.40.+j, 05.70.Ln

\section{INTRODUCTION}

After ten years of research and countless papers, the precise significance of self-organized criticality (SOC) [1] is still controversial. Originally, SOC was presented as a general theory to understand fractals and $1 / f$ noise as the natural outcomes of the dynamical evolution of systems with many coupled degrees of freedom. Irreversible dynamics would generate a self-organization of the system into a critical state, without the fine tuning of external parameters. The SOC idea was illustrated by computer models in which a slow external driving leads to a stationary state with avalanches of widely distributed amplitude [1]. This proposal stimulated a cascade of research activity in experiments, theory, and simulations. While the explanation presented in Ref. [1] about the origin of scaling in nature now appears too simplistic, SOC gave a formidable input to the study of slowly driven systems and avalanche phenomena.

Avalanche behavior was experimentally observed in a variety of phenomena ranging from magnetic systems (the Barkhausen effect) [2] and flux lines in high- $T_{c}$ superconductors [3], fluid flow through porous media [4], microfracturing processes [5], earthquakes [6], and lung inflation [7]. In addition, SOC ideas stimulated a great interest in granular matter [8], although it was soon realized that the concept was hardly applicable there, apart from the academic example of a rice pile [9]. All the above mentioned experiments share with SOC models the slow external driving and the avalanche response, but it is unclear whether self-organization as described in Ref. [1] plays any role there. To answer this question it would be necessary to understand better what determines the appearance of scaling in SOC models and driven systems in general.

The idea of a critical point without fine tuning of external parameters is very appealing, because it opposes the standard picture of equilibrium critical phenomena. The concept of "spontaneous" criticality, as it has been discussed in the literature, presents several ambiguities. It has been pointed out by several authors that the driving rate is a parameter that has to be fine tuned to zero in order to observe criticality [10-13]. This fact poses no problems to computer simulations, where an infinite time scale separation can easily be enforced, but it is crucial in experiments where the driving rate is always nonzero. The second ambiguity is mostly a language problem: calling "self-organization" the evolution toward a stationary state can be misleading. Any nonequilibrium system poised at its "fine tuned" critical point, when started from a generic configuration, evolves toward the critical stationary state, thus building up correlations and scaling. We would not describe this process as self-organization. These ambiguities in the definition of SOC have hindered the formulation of precise relations with other nonequilibrium critical phenomena [14].

In the past years, several attempts have been made to find a general mechanism to describe SOC models. In particular, Sornette and co-workers proposed several different mechanisms that could lead to SOC, or more generally to power law avalanche distributions $[15,16]$. In a recent paper, it was claimed that SOC corresponds to the tuning to zero of the order parameter of an ordinary critical phenomenon [11]. 
Our analysis shows that criticality arises from the fine tuning to zero of one or more control parameters (driving rate, dissipation) and there is no coupling between control and order parameters [13], in contrast with what was suggested in Ref. [15]. The incorrect identification of control and order parameters is at the basis of many misconceptions about SOC phenomena, as we will discuss in the following.

Many theoretical methods have been used in the analysis of SOC models. Few rigorous milestones can be found in the activity of Dhar and co-workers [17,18], and in the work of Ref. [19]. Flory [20] and Langevin-type approaches [21-23] have been used on a phenomenological basis. More recently, a real space renormalization group method provided good estimates of the avalanche exponents [12,24]. Despite their richness, however, all these approaches are focused on the critical avalanche behavior, and the external driving does not play any role; i.e., the system is studied in the infinite time scale separation regime. Furthermore, many of these attempts are conceived ad hoc for particular models, and do not provide a general conceptual framework to understand SOC phenomena.

The first step towards a comprehensive theoretical understanding of SOC is provided by mean-field (MF) theory, which gives insight into the fundamental physical mechanisms of the problem and a reference language. It provides a feasible treatment to nonequilibrium and complex problems (often the only one), and can be used as a starting point for more sophisticated calculations. Whereas many numerical and analytical approaches become harder as the dimensionality increases, MF theory improves and, despite crude approximations, it usually gives correct qualitative predictions for the phase diagrams of high-dimensional systems. Finally, MF theory highlights the importance of symmetries and conservation laws.

A vast activity concerning MF theory of SOC models can be found in the literature. Exponents describing avalanche distributions and propagation have been computed in several ways: solving infinite-range [25], Bethe lattice [18] and random neighbor [26-28] models, and by mapping the dynamics into a branching process [29-33]. Self-consistent MF approximations for the sand height distribution have also been used [20,34]. Other MF approaches use analogies with equilibrium critical phenomena [35,36], leading sometimes to incorrect predictions, as we will discuss in the following. In summary, the MF approach to SOC systems is composed of a number of studies of specific models, but a comprehensive understanding of the phenomenon is missing.

Here we present a unified MF description of SOC models using the formalism developed for nonequilibrium critical phenomena with steady states. We use a single site approximation to the master equation, and we enforce conservation laws by effective parameters and constraints. We concentrate on models driven by stochastic noise, such as the sandpile [1] and forest fire (FF) [37]. In order to write a master equation, we consider finite values of the driving rates, since only in this case are dynamical rules local in space and time. Our analysis shows that criticality in these models corresponds to the limit in which the dynamical rules become nonlocal. Nonlocality is implicitly enforced in computer simulations, where the evolution of a single site depends on the state of the entire system. This fact is particularly evident in extremal models in quenched disorder, where the dynamics proceeds by a global minimum search [38]. Also in that case, to write a local master equation one has to introduce a nonzero driving rate, but the driving mechanism differs from the one we discuss here. It is possible to relate these models to a corresponding nondriven critical phase transition [39].

The present approach allows us to identify control and order parameters of SOC models, and to clarify the relations with other nonequilibrium critical phenomena [13]. In particular, we show that SOC models have close similarities to nonequilibrium cellular automata with many absorbing states [40-42]. The major difference is that in SOC models, the control parameters have to be tuned to zero to reach criticality. As we discussed before, this limit corresponds to the breakdown of locality in the dynamical rules of the model, and hence to the onset of long-range correlation in the dynamical response. While apparently there is no mathematical difference between tuning a parameter to zero or to a nonzero value, the physical differences are quite important [10]. In the first case, changing the value of the control parameter by a given factor still keeps the system close to the critical point. This is not the case in ordinary phase transitions, where, doubling the value of the temperature, the system completely loses the critical properties. Moreover, in order for the SOC model to be defined, the control parameter (i.e., the driving rate) should always be nonzero, and the critical point can only be reached through a limit process.

The present MF theory can be applied to any stochastic cellular automaton, and therefore provides a unified description for the ensemble of SOC models and other related nonequilibrium critical systems, such as contact processes and cellular automata with absorbing states [40-42]. Moreover, it serves to emphasize the differences between different models and between different regimes in the same model. Our analysis also points out the inconsistencies contained in earlier MF approaches [35,36] which led to a misleading characterization of the model. We identify subcritical and supercritical states of SOC models, and discuss the different ways in which criticality can be reached. We describe the avalanche behavior characteristic of these models in terms of response functions, and study the effect of perturbations on the stationary state. We introduce a full set of critical exponents, describing the response at the critical point and the scaling close to the critical point in the subcritical and supercritical states. In the case of sandpile models, a subset of exponents is found to have mean-field values in any dimension. The reason for this behavior, which is confirmed by numerical simulations, is ascribed to the presence of conservation laws in the dynamics.

The paper is organized as follows: In Sec. II, we introduce the models. In Sec. III, we review the dynamic mean-field approximation to the master equation. Section IV contains the mean-field theory for the sandpile model, and discusses some issues related to conservation. In Sec. V, we report the mean-field analysis for the FF model, and Sec. VI is devoted to a general discussion. A brief report of these results appeared in Ref. [13].

\section{MODELS}

A rapid look at the SOC literature discourages every newcomer in the field. In less than ten years, more than 2000 
papers have been published, and comprehensive reviews are not yet appeared (a valuable effort in this direction can be found in Ref. [10]). This is due to the lack of a general understanding which would provide the framework to order the huge amount of information about SOC. In particular, we were spectators of an hectic activity in numerical simulations, with the introduction of a multitude of different models. A closer look at the literature reveals that the number of original models can be greatly reduced by noting that most of them are variations of prototype models. Using a more "Draconian" approach, we can distinguish just two main families of SOC models. The first is represented by stochastic SOC models such as the sandpile or forest-fire model, in which the self-organization process is the output of a stochastic dynamics. The second family groups together the socalled "extremal" or "quenched" models [38], which are defined by a deterministic dynamics in a random environment. Examples of the latter family are the invasion percolation [43] and bak-sneppen [44] models. In this paper we discuss stochastic models, but work is in progress to extend the present analysis to systems driven by an extremal dynamics.

\section{A. Sandpile models}

Sandpile models are cellular automata (CA) with an integer (or continuous) variable $z_{i}$ (energy) defined in a $d$-dimensional lattice. At each time step an energy grain is added to a randomly chosen site, until the energy of a site reaches a threshold $z_{c}$. When this happens the site relaxes,

$$
z_{i} \rightarrow z_{i}-z_{c},
$$

and the energy is transferred to the nearest neighbors,

$$
z_{j} \rightarrow z_{j}+y_{j}
$$

The relaxation of a site can induce nearest neighbor sites to relax on their turn, i.e., they exceed the threshold because of the energy received. New active sites can generate other relaxations and so on, eventually giving rise to an avalanche. For conservative models the transferred energy equals the energy lost by the relaxing site $\left(\Sigma y_{j}=z_{c}\right)$, at least on average. Usually, the only form of dissipation occurs at the boundary, from which energy can leave the system. It is worth remarking that during the avalanches, the energy input stops, until the system is again in equilibrium and no active sites are present. This corresponds to an infinite time scale separation. With these conditions the system reaches a stationary state characterized by avalanches whose sizes $s$ are distributed as a power law [1,45-48]

$$
P(s) \sim s^{-\tau} .
$$

The model originally introduced by Bak, Tang, and Wiesenfeld (BTW) [1] is a discrete automaton in which $z_{c}=2 d$ and $y_{j}=1$. An interesting variation of the original sandpile is the three-state Manna model [49]. In this automaton the critical threshold is $z_{c}=2$, fixed independently of the dimensionality $d$, and, if a relaxation (toppling) takes place, the energy is distributed into two randomly chosen nearest neighbor sites. Other variations in which part of the energy is kept by the relaxing site can also be considered, as well as directed mod- els in which energy is transferred along a preferential direction [45]. Finally, sandpile models that include a relaxation dynamics where part of the energy is dissipated have been considered [50]. These models can be characterized by the fraction of energy that disappears from the system during each relaxation process. When a global dissipation is present (energy is lost on average), the critical behavior is destroyed and a characteristic length is introduced. This numerical evidence suggests that conservation is necessary to obtain criticality.

As we discussed above, sandpile models are driven by adding a single energy grain on a randomly chosen site, when no active site is present. In this way, avalanches are instantaneous with respect to the driving time scale. This rule is very naturally implemented in a computer algorithm, which can handle the two different time scales at the same time. This, however, corresponds to a nonlocal interaction in which the site dynamical evolution depends upon the whole system configuration. This nonlocal interaction is hard to describe, and in order to perform an analytical description we have to fix a reference time scale, as for example the single site relaxation step, and measure the driving rate on that scale. For this reason, we consider a generalized sandpile model [35], that includes a nonvanishing driving rate, by introducing the probability $h$ per unit time that a site will receive a grain of energy. Energy is distributed homogeneously, and the total energy flux is given by $J_{\text {in }}=h L^{d}$. The parameter $h$ sets the driving time scale, or, equivalently, the typical waiting time between different avalanches, as $\tau_{d}$ $\sim 1 / h$. In the limit $h \rightarrow 0$, we recover the slow driving limit; i.e., during an avalanche the system does not receive energy. This formulation of the dynamics has the advantage of being local in space and time. The state of a single site depends only on the state of the site itself and its nearest-neighbor sites at the previous time step, through a transition probability that is given by the reaction and driving rates. It will be also convenient in the ensuing analysis to group the possible states of a site in three classes: active when $z \geqslant z_{c}$, critical when $z=z_{c}-1$, and stable for $z<z_{c}-1$.

\section{B. Forest-fire model}

The first example of a stochastic SOC model without conservation is in the FF model [37]. The model was first introduced in Ref. [51] as an example of SOC, and was then modified by Drossel and Schwabl [52]. The model is defined on a lattice in which each site can be empty, occupied by a green tree or a burning tree. Burning trees turn to ashes with a unitary rate, and set fire to the nearest neighbor trees. The model was first studied in the case of a small tree growth rate $p$, and in the absence of a spontaneous ignition of fires. In $d=2$, the system reaches a dynamical state in which fire fronts propagate with trivial scaling properties [53]. Only recently, large scale simulations have shown that for $d>2$ anomalous scaling laws occur [54]. A more interesting situation appears when a very small rate for spontaneous fire ignition $f$ (lightning probability) is introduced in the automaton dynamics. The system shows scaling behavior with a diverging characteristic length in the limit $f / p \rightarrow 0$ and $p$ $\rightarrow 0$, and the activity occurs in bursts of fire spreading (avalanches) whose distribution follows a power law behavior 
$P(s) \sim s^{-\tau}$. In the FF model, the driving rates are explicitly defined by $f$ and $p$, and the dynamical rules are thus local. However, in numerical simulations, the two driving fields are implicitly set to zero by the condition that tree growth and fire ignition occur only when the system does not show active sites. Only the ratio $\theta=f / p$ is quantitatively defined by the relative probability of tree growth with respect to fire ignition events. Also in this case, numerical simulations are done in the infinite time scale separation limit, which corresponds to the subcritical state of the system.

It is interesting to note the similarity between SOC models and nonequilibrium lattice automata with multiple adsorbing states [40]. These models present a critical phase transition separating two regimes: above the transition there is a finite density of active sites, while below the transition point this density is zero and the system freezes in one of the many stable configurations. In the following, using the formalism developed for this class of models, we will make this analogy more precise.

\section{DYNAMIC MEAN-FIELD APPROXIMATION}

In Sec. II, we generalized the definition of SOC automata to the fast-driving regime, thus removing the assumption of time scale separation commonly employed in simulations. This will turn out to be particularly convenient, since the restored locality of the dynamical rules allows a simpler description of the models.

The most generic description of SOC models is through a $d$-dimensional stochastic cellular automaton with $N=L^{d}$ sites, where $L$ is the lattice size. Each site $i$ on the lattice is characterized by an occupation variable $\sigma_{i}$ which can assume $q$ different values: for instance, the possible energy levels $z_{i}$ or the three different FF model states. The complete set $\sigma$ $=\left\{\sigma_{i}\right\}$ of lattice variables specifies a configuration of the system. The dynamical evolution of the system is determined by the transition probability $W\left(\sigma \mid \sigma^{\prime}\right)$ from the configuration $\sigma^{\prime}$ to the configuration $\sigma$. At each time step the state of a given site depends only on the previous state of the site itself and the set of sites interacting with it. The most general transition probabilities in the homogeneous and symmetric case is

$$
W\left(\sigma \mid \sigma^{\prime}\right)=\prod_{i=1}^{N} w\left(\sigma_{i} \mid \sigma^{\prime}\right)
$$

where $w\left(\sigma_{i} \mid \sigma^{\prime}\right)$ is the one site transition probability that depends on driving and reaction rates. The single site transition probability should satisfy the normalization property

$$
\sum_{\sigma_{i}} w\left(\sigma_{i} \mid \sigma^{\prime}\right)=1
$$

Because of the intrinsic nonequilibrium behavior of these systems, we have to consider the time-dependent probability distribution $P(\sigma, t)$ to have a configuration $\sigma$ at time $t$. From this distribution we can compute the average value of any function of the state $A(\sigma)$

$$
\langle A(t)\rangle=\sum_{\sigma} A(\sigma) P(\sigma, t) .
$$

The time evolution of the probability distribution is governed by the master equation (ME), which in continuous time reads

$$
\frac{\partial}{\partial t} P(\sigma, t)=\sum_{\sigma^{\prime}} W\left(\sigma \mid \sigma^{\prime}\right) P\left(\sigma^{\prime}, t\right)-W\left(\sigma^{\prime} \mid \sigma\right) P(\sigma, t) .
$$

The specific form of $W$ determines the dynamics of the model and the steady state distribution. Typically, SOC systems show a stationary state in which all the single time averages are time independent. To this state corresponds a stationary probability distribution $P(\sigma)=P(\sigma, t \rightarrow \infty)$. For equilibrium systems the stationary distribution has the Gibbs form $P(\sigma) \sim \exp (-\beta H(\sigma))$, where $H(\sigma)$ is the Hamiltonian. For SOC systems, like other nonequilibrium systems, there is not such a general criterion, but we have to solve the ME explicitly in the stationary limit. In practice, this is a formidable task which is accomplished just in very few cases. It is then necessary to use approximate methods in order to describe the collective behavior of these systems. The simplest available method is the dynamic cluster variation approach, which involves a hierarchy of evolution equations for the probability distribution of configuration of cluster of $k$ sites: $P_{k}\left(\sigma_{1}, \ldots, \sigma_{k}\right)$. If the system is homogeneous, the distribution of cluster of $k$ sites will be position independent. It is easy to recognize that $P_{1}$ represents the average density of sites in a certain state, while $P_{k>1}$ characterizes the correlation properties of the systems. Unfortunately, the time evolution equations for each $P_{k}$ depends on the higher correlation functions: the dynamical equation for the average densities depends on the two point correlation functions, the two point correlations on the three point correlations, and so on. Therefore, we have an infinite chain of coupled equations. The dynamical mean-field approximation consists of neglecting correlations up to a certain order. In the $n$-site approximation cluster probabilities are decoupled as a product of $n$-sites probabilities. This approximation has proved to be quite instructive for a qualitative description of the critical behavior of nonequilibrium systems [41].

Before proceeding to discuss in detail the single-site MF approximation for stochastic SOC models, we first discuss the basic symmetries of these systems, which will play a fundamental role in formulating a common description. We can reduce the number of states each site in the system can assume, noting that we can always identify three main states: stable $\left(\sigma_{i}=s\right)$, critical $\left(\sigma_{i}=c\right)$, and active $\left(\sigma_{i}=a\right)$. Stable sites are those that do not relax (become active) if energy is added to them by external fields or interactions with active sites. Critical sites become active with the addition of energy. Active sites are those transferring energy; they interact with other sites (usually nearest neighbors). Indeed, SOC always refers to systems in which the only state that generates dynamical evolution is the active one; i.e., stable and critical sites can change their state only because of external fields or by interacting with an active nearest neighbor. Therefore, SOC models correspond to three CA on $d$-dimensional lattices. This description is only approximate, since a certain amount of information is lost in grouping together stable sites. For instance, in the BTW model [1], we have several energy levels which pertain to a stable site, but 
we can take this fact into account, introducing some effective parameters in the ME. The three state description is exact for the Manna model [49] and the FF model [37].

In the simple MF single-site approximation, we denote by $\rho_{a}, \rho_{c}$, and $\rho_{s}$ the average densities of sites in the active, critical, and stable states, respectively. In the case of homogeneous systems, these densities can be written as

$$
\rho_{\kappa}(t)=\sum_{\{\sigma\}} \delta\left(\sigma_{j}-\kappa\right) P(\sigma, t) .
$$

The dynamical equations for the average densities are obtained from the ME by using Eq. (4),

$$
\begin{aligned}
\frac{\partial}{\partial t} \rho_{\kappa}(t)= & \sum_{\left\{\sigma^{\prime}\right\}} \sum_{\{\sigma\}} \delta\left(\sigma_{j}-\kappa\right)\left(\prod_{i} w\left(\sigma_{i} \mid \sigma^{\prime}\right) P\left(\sigma^{\prime}, t\right)\right. \\
& \left.-\prod_{i} w\left(\sigma_{i}^{\prime} \mid \sigma\right) P(\sigma, t)\right)
\end{aligned}
$$

The above equation can be simplified by using the normalization condition for the transition probabilities:

$$
\begin{gathered}
\sum_{\left\{\sigma^{\prime}\right\}} \prod_{i} w\left(\sigma_{i}^{\prime} \mid \sigma\right)=1 \\
\sum_{\{\sigma\}} \delta\left(\sigma_{j}-\kappa\right) \prod_{i} w\left(\sigma_{i} \mid \sigma^{\prime}\right)=w\left(\sigma_{j}=\kappa \mid \sigma^{\prime}\right) .
\end{gathered}
$$

Equation (9) can be further simplified when the interactions are only among a finite set of sites. In this case, with $\sigma^{\prime}$ $=\left\{\sigma_{i}^{\prime}, \sigma_{i+e}^{\prime}\right\}$ we denote the site $i$ and the set of sites that can interact with it-usually a finite number of sites or more commonly just the nearest neighbors (NN's). By restricting the sum and dropping the site index, because of the homogeneity, we finally obtain

$$
\frac{\partial}{\partial t} \rho_{\kappa}(t)=\sum_{\left\{\sigma^{\prime}\right\}} w\left(\kappa \mid \sigma^{\prime}\right) P\left(\sigma^{\prime}, t\right)-\rho_{\kappa}(t) .
$$

It is worth remarking that in the above expression the set $\sigma^{\prime}=\left\{\sigma_{i}^{\prime}, \sigma_{i+e}^{\prime}\right\}$ refers to the generic set of interacting sites which depends upon the particular dynamical rules and lattice geometry. In presence of a nonlocal interaction, the set $\sigma^{\prime}$ can correspond to the entire system. This presents a very difficult problem that can be treated, introducing a suitable regularization.

In general, therefore, we have that the evolution equations of the average densities are still coupled to the probability distribution of configurations of a set of interacting sites. In order to have a set of closed equations for the densities, we truncate the evolution equations by using a dynamical MF single site approximation. In this MF scheme we approximate the probability of each configuration $\sigma$ as the product measure of single site probabilities

$$
P(\sigma)=\prod_{i} P\left(\sigma_{i}\right) \equiv \prod_{i} \rho_{\sigma_{i}},
$$

thus neglecting all correlations in $P(\sigma)$. Introducing this approximation in Eq. (9), we obtain the MF reaction rate equations which depend just on the single site densities, and can be symbolically written as

$$
\frac{\partial}{\partial t} \rho_{\kappa}=F_{\kappa}\left(\rho_{a}, \rho_{c}, \rho_{s}\right), \quad \kappa=a, c, s,
$$

where $F_{\kappa}$ depends upon driving fields and interactions parameters through the transition rates $w$. In addition, because the densities must preserve normalization, two of the above equations supplemented with the condition $\rho_{a}+\rho_{c}+\rho_{s}=1$, are enough to describe the system completely.

In practice, the form of the rate equations depends upon the specific model. Nevertheless, we can write the general structure of the equations describing SOC models by simple considerations. In general, $F_{\kappa}$ can be expanded as a series of the average densities:

$$
F_{\kappa}=\sum_{n} f_{\kappa}^{n} \rho_{n}+\sum_{n, \ell} f_{\kappa}^{n, \ell} \rho_{n} \rho_{\ell}+O\left(\rho_{n}^{3}\right)
$$

where the constant term is set to zero in order to obtain a stationary state. The first order terms are the transition rates generated by the external driving fields or by spontaneous transitions. The second and higher order terms characterize transitions due to the interaction between different sites. In SOC models, only the active state generates a nontrivial dynamical evolution, while stable or critical sites can change their state only because of the external field or the presence of an active NN site. Since the critical point is identified by $\rho_{a}=0$, in correspondence with a vanishing external field, we can neglect second order terms in the density of active sites. The solutions of the stationary equations $\left[(\partial / \partial t) \rho_{\kappa}=0\right]$ are function of the effective parameters $f_{\kappa}^{n}$ and $f_{\kappa}^{n, \ell}$, which depend on the details of the model. It is expected that the critical behavior is not affected by the specific values of the parameters, while universality classes will depend on constraints imposed on the equations because of symmetries and conservation laws.

\section{MEAN-FIELD ANALYSIS OF SANDPILE MODELS}

Here we consider the explicit application of the single site MF approximation to the class of sandpile models. A simpler derivation based on symmetry considerations can be found in Ref. [13]. In Sec. III, we showed that the MF dynamical equations reduce in this approximation to the expression

$$
\frac{\partial}{\partial t} \rho_{\kappa}(t)=\sum_{\left\{\sigma^{\prime}\right\}} w\left(\kappa \mid \sigma^{\prime}\right) \prod_{i} \rho_{\sigma_{i}^{\prime}}(t)-\rho_{\kappa}(t),
$$

where $\sigma^{\prime}$ denotes the set formed by a single site and its set of interacting sites as specified by the dynamical rules. All the dynamical information of the system is contained in the transition rates $w\left(\kappa \mid \sigma^{\prime}\right)$. Unfortunately, the sandpile model is inherently nonlocal because of the implicit time scale separation. A site can receive energy only if the system is quescient. This implies that transition rates depend upon the whole set of lattice variables present in the system, giving rise to a strongly nonlocal dynamical rule. To have any hope 
to treat the model analytically, we have to assume that the sandpile dynamics can be realized as a limiting case of a model with local stochastic dynamics which respects the same symmetries and conservation laws as those of the original model. We refer to this as regularizing the sandpile rules by a suitable parametrization which allows us to recover the nonlocality in some particular limit. The simplest regularization was discussed in Sec. II, introducing the external flow of energy added to the system. We describe this external flow by the probability per unit time $h$ for a site to receive a grain of energy. The transition rates are now local, depending only on the field $h$ and the state of the nearest neighbor sites $\left(\sigma_{N N}\right)$ that determine the toppling dynamics. The total amount of energy added to the system at each time step will be $J_{\text {in }}=h L^{d}$ [55]. The nonlocality of the dynamical rules is recovered in the limit $h \rightarrow 0$ (see Sec. VI), that corresponds to an infinite time scale separation. The external field $h$ was historically introduced in Ref. [35]. Unfortunately, these early papers failed to address consistently the role played by driving and conservation, and led to several inconsistencies (see Sec. IV B). We limit our discussion to the present regularization of the sandpile dynamics for reasons of simplicity. Nevertheless, a more accurate characterization of the degree of nonlocality actually present in the infinitely slowly driven sandpiles can be obtained via more refined regularization schemes [56].

Since locality is restored $\left(\sigma^{\prime}=\left\{\sigma_{i}^{\prime}, \sigma_{\mathrm{NN}}^{\prime}\right\}\right)$, we can derive the MF equations for the density of active sites by considering the leading order in $h$ and $\rho_{a}$ in Eq. (16). The transition rates obey $w\left(a \mid a, \sigma_{\mathrm{NN}}\right)=0$, because an active site always transfers its energy, thus becoming stable at the next time step independently from its NN sites. In this way, we are neglecting higher contribution due to the presence of multiple active NN sites, which can transfer energy to the active site sustaining its activity. The only allowed transitions to the active state are due to critical sites which receive energy from the external driving or from active $\mathrm{NN}$ sites. In the absence of active NN sites, we have $w\left(a \mid c, \sigma_{\mathrm{NN}} \neq a\right)=h$. We can then obtain the contribution to the dynamical MF equation

$$
\sum_{\left\{\sigma_{\mathrm{NN}}^{\prime}\right\}} w\left(a \mid c, \sigma_{\mathrm{NN}}^{\prime} \neq a\right) \rho_{c} \prod_{i \in \mathrm{NN}} \rho_{\sigma_{i}^{\prime}}(t)=h \rho_{c}\left(1-\rho_{a}\right)^{Z}
$$

where $Z$ represents the lattice coordination number, i.e., the number of NN sites. If the sites do not receive energy from outside, we have to consider the possibilities that one of the $\mathrm{NN}$ sites is active and transfers energy to it. This process corresponds to

$$
w\left(a \mid c, \sigma_{i}=a, \sigma_{j \neq i} \neq a\right)=(1-h) \frac{g}{Z}(1-\tilde{p}),
$$

where $i, j \in \mathrm{NN}$. The right term represents the probability that a critical site receive an energy grain only from an active $\mathrm{NN}$. This is equal to the ratio between the number of sites $g$ involved in the dynamical relaxation process and the total number of NN's. For instance, $g=2 d$ for the BTW model [1] or $g=2$ for the Manna model [49]. In addition, we have to consider the probability $\tilde{p}$ that the active site does not transfer its energy because of intrinsic dissipation or because it is a boundary site. The above transition rate is valid only for homogeneous processes, and therefore excludes directed models. The total contribution due to this process, considering the multiplicity of active NN sites, is given by

$$
\begin{aligned}
\sum_{\left\{\sigma_{\mathrm{NN}}^{\prime}\right\}} w\left(a \mid c, \sigma_{i}^{\prime}\right. & \left.=a, \sigma_{j \neq i}^{\prime} \neq a\right) \rho_{c} \rho_{a} \prod_{j \neq i \in \mathrm{NN}} \rho_{\sigma_{j}^{\prime}}(t) \\
& =(g-\epsilon) \rho_{c} \rho_{a}(1-h)\left(1-\rho_{a}\right)^{Z-1},
\end{aligned}
$$

where the parameter $\epsilon$ conveniently identifies the average energy dissipated $g \tilde{p}$ in each elementary process. It is worth remarking that $\epsilon$ is also present for fully conservative systems, being an effective term due to the boundary dissipation: it acts as an external tunable parameter in the case of bulk dissipation and accounts for size effects in finite systems.

Neglecting higher orders in $h$ and $\rho_{a}$ from the Eqs. (17) and (19), we can finally write the MF dynamical equation for the densities of active sites:

$$
\begin{aligned}
\frac{\partial}{\partial t} \rho_{a}(t)= & -\rho_{a}(t)+h \rho_{c}(t)+(g-\epsilon) \rho_{c}(t) \rho_{a}(t) \\
& +\mathcal{O}\left(h \rho_{a}, \rho_{a}^{2}\right) .
\end{aligned}
$$

Next we derive the dynamical MF equation for the density of stable sites, following the same strategy used above. Since, at lowest order, active sites become stable with unitary rate, we have that $w\left(s \mid a, \sigma_{\mathrm{NN}}^{\prime}\right)=1+\mathcal{O}\left(h \rho_{a}, \rho_{a}^{2}\right)$, yielding a contribution to the MF equation which is

$$
\sum_{\left\{\sigma_{\mathrm{NN}}^{\prime}\right\}} w\left(s \mid a, \sigma_{\mathrm{NN}}^{\prime}\right) \rho_{a} \prod_{i \in \mathrm{NN}} \rho_{\sigma_{i}^{\prime}}(t)=\rho_{a}+\mathcal{O}\left(h \rho_{a}, \rho_{a}^{2}\right)
$$

Since critical sites never become stable, we have also that $w\left(s \mid c, \sigma_{\mathrm{NN}}^{\prime}\right)=0$.

Energy conservation imposes that energy is stored in stable sites until they become critical. This implies a nonunitary rate $w\left(s \mid s, \sigma_{\mathrm{NN}}^{\prime}\right)$. The simplest way to derive this term makes use of the normalization condition that yields $w\left(s \mid s, \sigma_{\mathrm{NN}}^{\prime}\right)=1-w\left(c \mid s, \sigma_{\mathrm{NN}}^{\prime}\right)$. In fact, these transition rates are nearly equivalent to those from critical to active sites. The only difference is that only a fraction $u$ of stable sites receiving an energy quantum will contribute to the $s \rightarrow c$ process, i.e., the fraction of stable sites which are subcritical. Therefore, the reaction rates are related by the factor $u$ as $w\left(c \mid s, \sigma_{\mathrm{NN}}^{\prime}\right)=u w\left(a \mid c, \sigma_{\mathrm{NN}}^{\prime}\right)$. Recalling the derivation of Eq. (20), it is straightforward to obtain

$$
\begin{aligned}
& \sum_{\left\{\sigma_{\mathrm{NN}}^{\prime}\right\}} {\left[1-w\left(c \mid s, \sigma_{\mathrm{NN}}^{\prime}\right)\right] \rho_{s} \prod_{i \in \mathrm{NN}} \rho_{\sigma_{i}^{\prime}}(t) } \\
& \quad=\rho_{s}-u h \rho_{s}-u(g-\epsilon) \rho_{s} \rho_{a}+\mathcal{O}\left(h \rho_{a}, \rho_{a}^{2}\right) .
\end{aligned}
$$

Adding all these contributions, we finally obtain the dynamical MF equation 


$$
\begin{aligned}
\frac{\partial}{\partial t} \rho_{s}(t)= & \rho_{a}(t)-u h \rho_{s}(t) \\
& -u(g-\epsilon) \rho_{s}(t) \rho_{a}(t)+\mathcal{O}\left(h \rho_{a}, \rho_{a}^{2}\right),
\end{aligned}
$$

that together with Eq. (20), and supplemented with the normalization condition, fully describes the MF evolution of sandpile automata.

In deriving the MF equations we have made an approximation, introducing the parameter $u$ to take into account the presence of several energy levels instead of a single stable level. For three level models $u=1$, and this description is exact, while for multilevel models like that of BTW [1] the parameter $u$ can be determined self-consistently in the stationary state using the energy conservation [13]. Here we show that we can also obtain $u$ by the full description of the MF equations. We consider a generic sandpile model in which the energy threshold is $z_{c}$, and, after a relaxation event, $g$ energy grains are transfered to randomly chosen neighbors. For instance, the $d$-dimensional BTW model has $z_{c}=2 d$ and $g=2 d$, but we can think these are arbitrary values for these parameters. We can describe these systems in more detail by introducing the densities $\rho_{n}$, describing the probability that a site is in the level $n$. We then have that $\rho_{c}=\rho_{z_{c}-1}$ and $\rho_{s}=\Sigma_{n=0}^{z_{c}-2} \rho_{n}$. The dynamical evolution can be simplified noting that an active site with energy $z_{c}$ becomes stable, and its energy becomes $n=z_{c}-g$. One can show that stable sites, with energy levels lower than $n$, have a zero stationary density. Without loss of generality, we can therefore assume that the zero energy level is $n=z_{c}-g$. By rescaling the energy levels in this way, we obtain $\rho_{c}=\rho_{g-1}$ and $\rho_{s}=\sum_{n=0}^{g-2} \rho_{n}$. The intermediate levels are described by the $\mathrm{MF}$ equation

$$
\begin{aligned}
\frac{\partial}{\partial t} \rho_{n}(t)= & -h \rho_{n}(t)-(g-\epsilon) \rho_{n}(t) \rho_{a}(t)+h \rho_{n-1}(t) \\
& +(g-\epsilon) \rho_{n-1}(t) \rho_{a}(t)+\mathcal{O}\left(h \rho_{a}, \rho_{a}^{2}\right),
\end{aligned}
$$

where $1 \leqslant n \leqslant g-2$. In the stationary state we obtain $\rho_{n}$ $=\rho_{n-1} \cdots=\rho_{0}$ and, noting that $u=\rho_{g-2} /\left(\sum_{n=0}^{g-2} \rho_{n}\right)$, we recover the result $u=1 /(g-1)$ obtained in Ref. [13]. This result expresses the energy conservation and fixes the stationary solution consistently with the energy balance. Noticeably, in the Manna model [49], for which $g=2$, we obtain $u=1$, as must be for a three state model. As a last remark we point out that, summing up the above set of equations with the one for $\rho_{0}$,

$$
\frac{\partial}{\partial t} \rho_{0}(t)=-h \rho_{0}(t)-(g-\epsilon) \rho_{0}(t) \rho_{a}(t)+\rho_{a}(t),
$$

we obtain Eq. (23) as a function of the parameter $u$.

When the system is far from the stationary state, the parameter $u$ will in general be time dependent. In the following, we will always consider stationary properties or homogeneous perturbations which leave $u$ unchanged, but we could think of situations in which $u$ does not have a stationary value. This corresponds to a systems kept far from its "natural" configuration. This can have strong influence even on the critical properties of the system as in the case of CA with absorbing states. These features will be discussed elsewhere [56].

To study the stationary MF solutions, we consider the simple three level case, and determine $u$ self-consistently as in Ref. [13]. Combining the normalization equation with the stationarity limit of Eqs. (20) and 23, we obtain the set of equations

$$
\begin{gathered}
\rho_{a}=h \rho_{c}+(g-\epsilon) \rho_{c} \rho_{a}, \\
\rho_{a}=u h \rho_{s}+u(g-\epsilon) \rho_{s} \rho_{a}, \\
\rho_{a}=1-\rho_{s}-\rho_{c} .
\end{gathered}
$$

After some algebra from Eqs. (26), we obtain a closed equation for $\rho_{a}$ :

$$
u(g-\epsilon) \rho_{a}^{2}+[1+u(1+h-g+\epsilon)] \rho_{a}-u h=0 .
$$

We can expand $\rho_{a}(h)$ for small values of the field $h$. The zero order term in the expansion vanishes, and we obtain a leading linear term

$$
\rho_{a}(h)=\frac{u h}{1+u(1-g+\epsilon)} .
$$

This result has to be consistent with the global conservation law, which states that the average input energy flux $J_{\text {in }}$ must balance the dissipated flux $J_{\text {out }}$. In the stationary state, the conservation law can be written as

$$
J_{\text {in }}=h L^{d}=J_{\text {out }}=\epsilon \rho_{a} L^{d} .
$$

By comparing Eqs. (28) and (29), we obtain that $u=1 /(g$ $-1)$, which is the result we previously obtained from the complete analysis. In the limit $h \rightarrow 0$ the densities are therefore given by

$$
\rho_{a}=\frac{h}{\epsilon}, \quad \rho_{c}=\frac{1}{g}+O(h), \quad \rho_{s}=\frac{g-1}{g}+O(h) .
$$

The numerical values for the density of critical and stable sites are nonuniversal quantities, and depend on the lattice geometry and dynamical rules of each specific model via the parameter $g$. The result for $\rho_{c}$ can be directly compared with the estimates from numerical simulations of several models by substituting the correct value of $g$. For the original BTW model ( $g=2 d$ ), extensive numerical simulations on the density of energy levels can be found in Ref. [34]. As expected, the agreement with the MF result increases for highdimensional systems, and we recover the exact result in the limit $d \rightarrow \infty$.

We next discuss the critical behavior of these systems. The balance between conservation laws and the dissipation are essential for the critical behavior of the model, as also pointed out in Ref. [33]. The model is critical just in the double limit $h, \epsilon \rightarrow 0, h / \epsilon \rightarrow 0$, similarly to the forest-fire model [37]. We are going to see that in this limit the zero field susceptibility of the system is singular, signaling a longrange (critical) response function. The onset of the critical behavior is then recovered in the limit of vanishing driving field corresponding to the locality breaking in the dynamical 


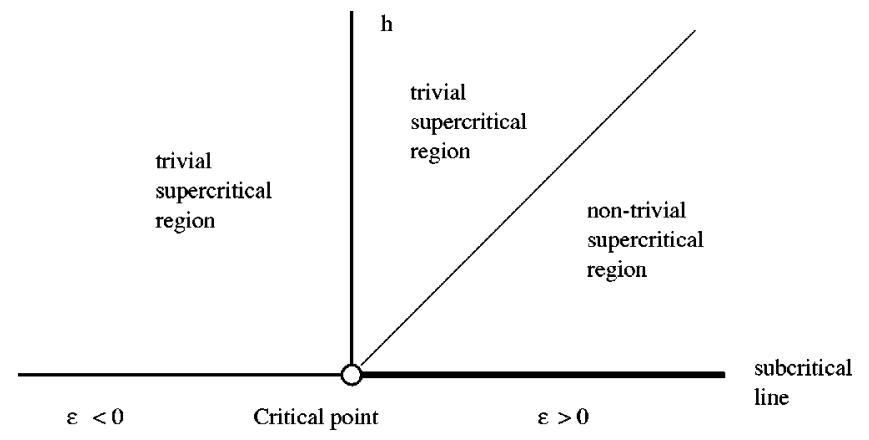

FIG. 1. Phase diagram of a generic sandpile model. We include negative values of the dissipation corresponding to a net addition of energy during the avalanches. The trivial supercritical regime is given by a saturation of the system, which is receiving more energy than it can dissipate. The interesting region $h<\epsilon$ is discussed in the text.

evolution. In analogy with nonequilibrium phenomena [40,42], the one particle density of active sites is the order parameter, and goes to zero at the critical point. The driving and dissipation rates identify the two control parameters, i.e., the relevant scaling fields. We can then distinguish different regimes as a function of the control parameters. The model is supercritical for $h>0$ and $\epsilon>h$, while for $h \rightarrow 0$ and $\epsilon>0$ it is subcritical and the dynamics displays avalanches. The phase diagram is somehow similar to that of usual continuous phase transitions, if we replace $h$ by the magnetic field and $\epsilon$ by the reduced temperature (see Fig. 1). We can fully exploit this analogy by allowing the parameter $\epsilon$ also to assume negative values. This corresponds to a sandpile in which a positive net amount of energy enters the system during the avalanche activity (negative dissipation). The resulting supercritical regime is analogous to many nonequilibrium systems with a negative reduced control parameter. However, for $h>\epsilon$, the system has only a trivial stationary state, since $\rho_{a}$ would have to be greater than 1 to satisfy Eq. (29). Thus, in the nontrivial supercritical region, $h$ and $\epsilon$ cannot be varied independently, because the global conservation imposes that $h<\epsilon$. This restricts the scaling behavior to particular limit values of the control parameters. In the following, we individuate the regimes corresponding to the standard sandpile numerical simulations and a scaling regime in the supercritical region of the phase space.

\section{A. Subcritical regime}

The standard numerical simulations of sandpile models are carried out in the presence of an infinite time scale separation. As already discussed in previous sections, this implies an infinitely slow driving of the systems, i.e., $h \rightarrow 0$. In this limit, there is a single control parameter, the intrinsic or boundary dissipation $\epsilon$, and the order parameter $\rho_{a}$ is identically zero in the steady state. To describe the critical behavior quantitatively, we study the effect of a small perturbation $\Delta h$ on the steady state density

$$
\Delta \rho_{a}(x, t)=\int \chi_{h, \epsilon}\left(x-x^{\prime} ; t-t^{\prime}\right) \Delta h\left(x^{\prime}, t^{\prime}\right) d x^{\prime} d t^{\prime},
$$

where $\chi_{h, \epsilon}\left(x-x^{\prime} ; t-t^{\prime}\right)$ is the response function of the sys- tem. We define the total susceptibility $\chi_{h, \epsilon}$ of the system as the integral over space and time of the response function, and show in Appendix A for the stationary state that

$$
\chi_{\epsilon} \equiv \lim _{h \rightarrow 0} \chi_{h, \epsilon}=\left.\frac{\partial \rho_{a}(h)}{\partial h}\right|_{h=0} .
$$

This immediately gives the zero field susceptibility

$$
\chi_{\epsilon}=\frac{1}{\epsilon},
$$

which diverges as $\epsilon \rightarrow 0$. The system is in a subcritical state for any value of $\epsilon$ different from zero. For $\epsilon=0$, the system reaches a critical point in which the response function becomes long ranged and the susceptibility diverges. Close to this critical point the scaling behavior is characterized by the scaling laws $\chi_{\epsilon} \sim \epsilon^{-\gamma}$, with $\gamma=1$, and by the divergence of the correlation length $\xi \sim \epsilon^{-\nu}$.

An important result can be derived from the response function by defining

$$
\chi_{\epsilon}(r)=\int \chi_{\epsilon}(r, t) d t
$$

as the average total response received at position $r$, when $\Delta h\left(x^{\prime}, t^{\prime}\right)=\delta\left(x^{\prime}\right)$. Since energy is transfered locally and isotropically, the net energy current is given by $j$ $\sim \partial \chi(r) / \partial r$. For locally conservative models the energy current $j$ must satisfy in average the conservation law

$$
\int j d \sigma=\text { const, }
$$

where $d \sigma$ is the $(d-1)$-dimensional surface element. This ensures that the energy flowing into the system is balanced by the dissipated energy in the stationary state. Hence, at large $r$, we have the solution $\chi(r) \sim r^{2-d}$. A similar result has been obtained in Ref. [20]. In the presence of boundary or intrinsic dissipation, the system acquires a finite correlation length, and we can establish the general scaling form

$$
\chi_{\epsilon}(r)=\frac{1}{r^{d-2}} \Gamma(r / \xi)
$$

where $\Gamma(r / \xi)$ is a cutoff function for $r \gg \xi$. This immediately gives the following relation between $\xi$ and the zero field susceptibility:

$$
\chi_{\epsilon}=\int \chi_{\epsilon}(r) r^{d-1} d r \sim \xi^{2} .
$$

We find the MF value of the correlation exponent $\nu=\frac{1}{2}$ by substituting $\xi \sim \epsilon^{-\nu}$, and comparing with Eq. (33).

We can use these exponents to characterize the finite size scaling of the conservative sandpile model, since our MF analysis treats both boundary and bulk dissipation in the same way. In conservative systems, when the size is increased the effective dissipation depends on the system size and we assume that $\epsilon \sim L^{-\mu}$. In fact, the dissipation rate is given by the probability to find a border site instead of a bulk 
site during an avalanche. Thus the exponent $\mu$ links the dissipation rate with the system finite size, providing a unified view of locally dissipative and open-boundary models. In the conservative case, the characteristic length of avalanches should go like $\xi \sim L$ to ensure dissipation of energy outside the boundaries. This implies the scaling relation $\nu \mu=1$, which immediately gives $\mu=2$. We show in Appendix A that the susceptibility scales as the average avalanche size

$$
\chi_{\epsilon} \sim\langle s\rangle,
$$

which implies $\chi \sim L^{\mu \gamma}$. From this result, we obtain the scaling law

$$
\langle s\rangle \sim L^{2} \quad \text { for } L \rightarrow \infty,
$$

which was found numerically in various dimensions [46], and rigorously proven in $d=2$ [17]. Our explanation implies that the diffusive behavior of the avalanches is due to the global conservation law.

Summarizing all these results, we obtain a first set of MF exponents:

$$
\gamma=1, \quad \mu=2, \quad \nu=\frac{1}{2} .
$$

In deriving these exponents we made only use of conservation laws. Therefore we expect that these values hold in all dimensions, and can be considered as exact results. In Sec. II B we will confirm these results by numerical simulations performed in the fast driving regime, and discuss some results already published in the literature.

In the subcritical regime the dynamics takes place in the form of avalanches, but if $h=0$ the system rapidly decays in one of the adsorbing configurations; the ones with no active sites. All of them are stable in the absence of a driving field. It is useful to characterize the proliferation of active sites starting from a seed initial condition. In close analogy with CA with adsorbing states, we study the spreading of active sites after a small perturbation. We prepare the system in an initial state consisting of a single active site, i.e., an infinitesimal perturbation in the driving field $\Delta h(x, t)=\delta(t) \delta(x)$. Since $h(t>0)=0$, active sites cannot be produced spontaneously from critical sites, and can only appear due to the spreading of the initial perturbation. The properties of this process close to the critical point characterize the avalanche behavior typical of SOC phenomena.

Following Grassberger and de la Torre [40], we consider the probability that a small perturbation activates $s$ sites (an avalanche in the SOC terminology)

$$
P(s, \epsilon)=s^{-\tau} \mathcal{G}\left(s / s_{c}(\epsilon)\right),
$$

where $s_{c} \sim \epsilon^{-1 / \sigma}$ is the cutoff in the avalanche size. The perturbation decays in the stationary subcritical state as

$$
\rho_{a}(t) \sim t^{\eta} \mathcal{F}\left(t / t_{c}(\epsilon)\right) .
$$

Here $t_{c}$ denotes the characteristic time which scales as $t_{c}$ $\sim \epsilon^{-\Delta}$. We can also introduce the scaling exponents which relate cutoff lengths to the characteristic size, $s_{c} \sim \xi^{D}$ and $t_{c} \sim \xi^{z}$. These exponents are related by the scaling laws

$$
D=\frac{1}{\nu \sigma}, \quad z \nu=\Delta .
$$

Another scaling relation between critical exponents can be obtained from Eq. (38) (see Appendix A),

$$
\chi_{\epsilon} \sim\langle s\rangle=\int s^{-\tau+1} \mathcal{G}\left(s / s_{c}(\boldsymbol{\epsilon})\right) d s \sim \boldsymbol{\epsilon}^{(\tau-2) / \sigma},
$$

which implies that

$$
\gamma=\frac{(2-\tau)}{\sigma} .
$$

To obtain the MF values of the avalanche exponents, we solve the evolution equation for a small perturbation close to the stationary state. We consider $\rho_{\kappa}(t)=\rho_{\kappa}+\delta \rho_{\kappa}(t)$, where $\delta \rho_{\kappa}(t)$ is the deviation of the densities from their stationary value. By considering a small perturbation around the stationary state, keeping only the linear term in $\delta \rho_{\kappa}(t)$, and using the normalization condition, we obtain

$$
\begin{aligned}
\frac{\partial}{\partial t} \delta \rho_{a}(t)= & -\delta \rho_{a}(t)+h \delta \rho_{c}(t)+(g-\epsilon) \rho_{c} \delta \rho_{a}(t) \\
& +(g-\epsilon) \rho_{a} \delta \rho_{c}(t), \\
\frac{\partial}{\partial t} \delta \rho_{s}(t)= & \delta \rho_{a}(t)-\frac{h}{g-1} \delta \rho_{s}(t)-\frac{g-\epsilon}{g-1} \rho_{s} \delta \rho_{a}(t) \\
- & \frac{g-\epsilon}{g-1} \rho_{a} \delta \rho_{s}(t), \\
& \delta \rho_{a}(t)=-\delta \rho_{c}(t)-\delta \rho_{s}(t) .
\end{aligned}
$$

In subcritical regimes $(h \rightarrow 0)$, we only keep in these equations the leading terms in $\epsilon$. Substituting in Eqs. (46) the densities given by the solution of the stationary equation for $h \rightarrow 0$ (i.e., $\rho_{a}=0$ and $\rho_{c}=1 / g$ ), we finally obtain the evolution equation in diagonal form:

$$
\frac{\partial}{\partial t} \delta \rho_{a}(t)=-\frac{\epsilon}{g} \delta \rho_{a}(t) .
$$

The solution of Eq. (47) is given by

$$
\delta \rho_{a}(t) \sim \exp (-\epsilon t / g),
$$

which implies $\eta=0$. The last equation also defines the characteristic relaxation time for an infinitesimal perturbation to be $t_{c}=g / \epsilon$, yielding $\Delta=1$. We compute the remaining exponents using a further scaling relation, which we derive in Appendix B:

$$
\frac{(\tau-1)}{\nu \sigma}=z
$$

It is worth remarking that Eq. (49) is valid only in MF theory. By combining these relations with those of Eq. (43), we obtain the second set of MF critical exponents:

$$
z=2, \quad D=4, \quad \tau=\frac{3}{2}, \quad \sigma=\frac{1}{2} .
$$

It is worthwhile to remark that the numerical value of these exponents is the same as in other MF approaches, but their significance is completely different, being defined with re- 
spect to a different scaling field. All sandpile models with the same dynamical MF equations share the same critical exponents and belong to the same universality class. However, the degree of universality is highly overstated, as usually happens in MF approaches. In particular, the exponents do not depend on the dimensionality $d$. The exponents describing avalanche distributions in low-dimensional systems, in general, will not agree with the results of MF theory. For instance, it is still controversial if the BTW and two state models are in the same universality class. While it was believed for some time that this was indeed the case, recently large scale numerical simulations questioned this statement [57].

To compute the value of critical exponents below the upper critical dimension, we have to use renormalization group techniques, which allow the correct treatment of the scalefree fluctuations present at the critical point. The renormalization group approach to a nonequilibrium system presents several difficulties which can be overcome by suitable approximations [12,24].

\section{B. Supercritical regime}

The supercritical region is characterized by a finite density of active sites, i.e., a nonzero order parameter. Close to the critical point, the supercritical region corresponds to the parameter ranges $h \ll 1, \epsilon \ll 1$ and $h \lesssim \epsilon$. In this regime the order parameter is linear in $h$,

$$
\rho_{a} \sim h^{1 / \delta}, \quad \delta=1,
$$

as we obtain from Eq. (30). The same result was also conjectured in Ref. [46]. This is analogous to the MF results obtained for contact processes and other nonequilibrium CA [40-42], but it is in contrast with previous MF approaches for sandpile models [35,36], which yielded $\delta=2$. This latter incorrect result is due to an inconsistency present in those studies. The scaling is expressed in terms of the average energy $\langle z\rangle \equiv \Sigma_{i} \rho_{i} z_{i}$, which is treated as an independent control parameter. As we have just shown, $\langle z\rangle$ and $h$ are not independent in the stationary state. The stationary probability distribution of heights $\rho_{i}$ is indeed a function of the driving rate. Moreover, $\langle z\rangle$ cannot be considered as the control parameter even for $h=0$, since it does not determine completely the state of the system: The same value of $\langle z\rangle$ describes several states corresponding to different values of densities $\rho_{i}$. This is a typical property of CA with multiple absorbing states [42]. In preparing an initial condition consisting of a localized active region, one has considerable freedom to choose the initial state (the adsorbing configuration). In order to observe the critical properties of the system, we should choose one of the "natural" initial configurations, which can be obtained by the dynamical evolution for infinitesimal driving in the long time limit. In numerical simulations this is equivalent to first preparing the system in the stationary state in the presence of the time scale separation, and then average over the many different realizations of the avalanches.

The exponent $\delta$ has been defined in previous works in analogy with usual continuous phase transitions, where it characterizes the scaling of the order parameter in the presence of an external field when the other critical parameters are set to zero. In SOC, however, $h$ and $\epsilon$ are not fully independent because of the $\epsilon>h$ condition. We can define a consistent scaling regime with respect to the reduced variable $\phi=h / \epsilon$ in the double limits $h \rightarrow 0$ and $\epsilon \rightarrow 0$, with the supplementary conditions that $\epsilon \ll \phi \ll 1$. These limits define a parameter region which is identified roughly as $\epsilon \ll 1$ and $\epsilon^{2} \ll h \ll \epsilon$. In this region, the MF approximations show that the order parameter is positive and scales as

$$
\rho_{a}=\phi^{\beta},
$$

with $\beta=1$. The exponent $\beta$ characterizes the scaling behavior of the order parameter with respect to the reduced parameter $\phi$, and should not be confused with the exponent $\delta$. In order to uncover the scaling of the characteristic lengths of the system with respect to the parameter $\phi$, we study the evolution of small perturbations around the stationary state. As in Sec. IV A, we denote by $\delta \rho_{\kappa}(t)$ the deviations from the stationary state, and write the dynamical equation keeping only the leading terms. We thus neglect in Eqs. (46) the terms in $h$ and $\epsilon$, and keep the terms in $\phi$. For this we compute the first order correction in $\phi$ to the values of the stationary densities. These results to be $\rho_{a}=\phi, \rho_{c}=(1$ $-\phi) / g$ and $\rho_{s}=(g-1)(1-\phi) / g$, and, by substituting in the dynamical MF equations, we obtain

$$
\begin{gathered}
\frac{\partial}{\partial t} \delta \rho_{a}(t)=-(g-1) \phi \delta \rho_{a}(t)-g \phi \delta \rho_{s}(t), \\
\frac{\partial}{\partial t} \delta \rho_{s}(t)=+\phi \delta \rho_{a}(t)-\frac{g}{g-1} \phi \delta \rho_{s}(t)
\end{gathered}
$$

By diagonalizing Eqs. (53), we find the eigenvalues

$$
\Lambda_{ \pm}=-\phi f_{ \pm}(g)
$$

where $f_{ \pm}(g)$ always has a positive real part. Both eigenvalues are thus negative and linear in $\phi$, and represent the inverse of the relaxation time scale of a perturbation around the stationary state. We have, therefore, that $t_{c} \sim \phi^{-\Delta^{\prime}}$, with $\Delta^{\prime}=1$, implying that the characteristic time scales as in the subcritical regime. The solution for the spreading of the density perturbation has the form $\delta \rho_{a}(t) \sim \mathcal{F}\left(t / t_{c}\right)$, yielding, as in the subcritical regime, $\eta^{\prime}=0$.

Equations (53) describe how a localized perturbation decays in the stationary state. As in Sec. IV A, this decay can be related to the susceptibility

$$
\chi_{\phi} \sim \int \delta \rho_{a}(t) d t \sim \phi^{-\gamma^{\prime}}
$$

with $\gamma^{\prime}=1$. A characteristic length $\xi$ is associated with the characteristic time of fluctuations. Since energy is transferred homogeneously and isotropically, we have that $\chi_{\phi} \sim \xi^{2}$, as in the subcritical regime. By comparing this relation with Eq. (55), we obtain $\xi^{2} \sim \phi^{-1}$, or $\xi \sim \phi^{-\nu^{\prime}}$ with $\nu^{\prime}=\frac{1}{2}$. We can obtain a clearer picture of this behavior using the avalanche representation. The condition that the time between two energy addictions (the driving time scale) is much longer than the fluctuation time scale can be written as 


$$
h \ll t_{c}^{-1} \sim \phi .
$$

This condition is implicitly verified in the limit we are considering $(h \ll \epsilon \ll \phi)$. Under this assumption, it is very unlikely for fluctuations to overlap. Thus, on average, each event is separated from the others, and can be defined as in the subcritical case as an avalanche. In the stationary state, the average nonzero order parameter is produced by the random appearance of a finite number of nonoverlapping avalanches. Furthermore, we can identify the response function with the time evolution of an avalanche, and we recover in the limit $h \rightarrow 0$ that $\chi_{\phi} \sim\langle s\rangle$. In its turn the latter implies that in this regime the average sizes of avalanches diverge as $\langle s\rangle \sim \phi^{-1}$ as $\phi \rightarrow 0$. Work is in progress to obtain a numerical confirmation of the MF predictions for the supercritical regime.

\section{Numerical simulations}

In this section, we compare the results of numerical simulations with the prediction of MF theory, and in particular we expect that the set of MF exponents related to the global conservative nature of the model are also valid in low dimensions. Sandpile models have been extensively studied only in the subcritical state [45-48]. Most of the numerical results refer to an avalanche distribution in the conservative limit, with open-boundary conditions. In these conditions the finite size scaling has been found to be problematic, and despite the use of very large scale simulations there is not complete agreement on the values of the exponents [48]. The reason for this is probably that open-boundary conditions impose a value for the effective dissipation which depends on the lattice size, and does not act homogeneously through the system.

We simulate the BTW model numerically with finite driving rate $h$ and boundary dissipation in $d=2$. In this case the dissipation is implicitly considered through the openboundary conditions. When a boundary site topples, it dissipate part of the energy outside, without transferring it to the neighbors. The driving rate $h$ is introduced as the probability for unit time that a site receives an energy grain. Apart from the driving the simulation proceeds as in Ref. [46]. We see in Fig. 2 that the density of critical sites goes to zero linearly with $h(\delta=1)$, with a slope that increases with the system size as $L^{2}$. This is in agreement with the MF theory which predicts that the susceptibility scales as $L^{\mu \gamma}$, with $\mu \gamma=2$.

To observe more clearly the scaling with dissipation of the sandpile model, we study the BTW model with periodic boundary conditions and fixed dissipation $\epsilon$. We model the dissipation introducing a probability $\tilde{p} \equiv \epsilon / g$, for which the energy in a relaxation event is lost, instead of being transfered. In Fig. 3, we plot the susceptibility $\chi_{\epsilon}=d \rho / d h$ as a function of $\epsilon$. We observe the $1 / \epsilon$ behavior $(\gamma=1)$ predicted by mean-field theory. We should add to this the value of $\nu$ $=0.5$ that was obtained studying a dissipative sandpile model in $d=2$ [50].

In summary, we have shown that some MF features are present in the $d=2$ sandpile model. The global conservation law imposes that the exponents $\mu, \nu$, and $\gamma$ assume their MF values. This strongly supports the MF picture provided here. The other critical exponents do not necessarily assume

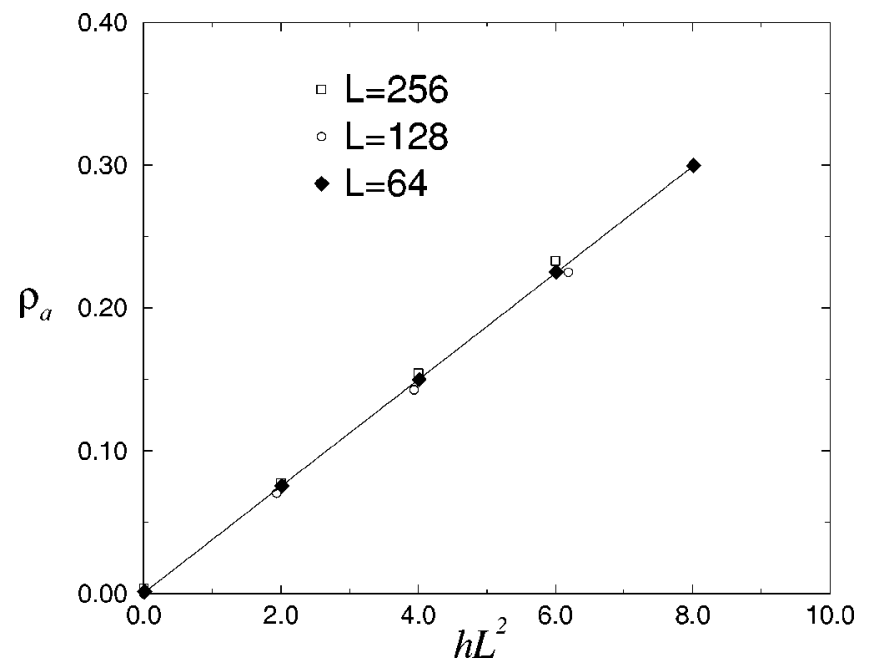

FIG. 2. The density of active sites in the BTW model with boundary dissipation, as a function of the driving rate $h$, is plotted for different system sizes $L$.

mean-field values in $d=2$, and we do not analyze them here. Extensive measurements of these exponents can be found in the literature [45-48]. We are currently performing simulations of the model in the supercritical regime in $d>2$, and the results will be published elsewhere [58].

A very important issue in SOC models is the definition of universality classes. We expect critical properties, such as exponents and scaling, to be independent of the precise values of dynamical parameters, being determined only by the symmetry of the problem and the number of spatial dimension. Therefore, it is important to understand to what extent we can change the automaton rules without changing the universality class. The dynamical rules, in fact, contain the basic symmetries of SOC models, and it would be interesting to classify the critical behavior in terms of their microscopic dynamics. So far, no theoretical treatment has been able to address this question rigorously. Also, from the point of view of numerical simulations, the situation is controversial. It

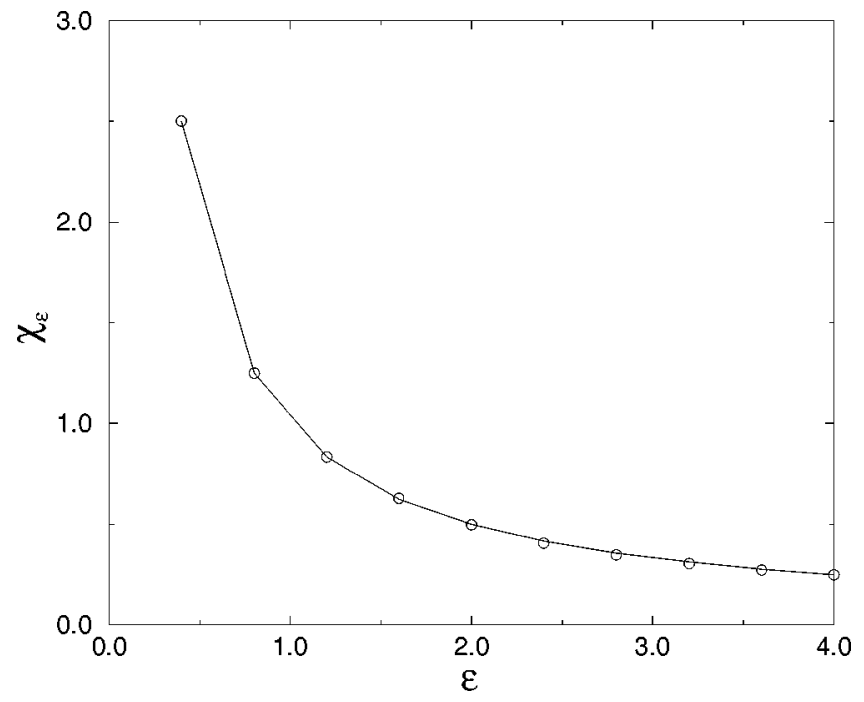

FIG. 3. The susceptibility $\chi_{\epsilon}=d \rho_{a} / d h$ as a function of the dissipation $\epsilon$, for a system with periodic boundary conditions and size $L=64$. The line corresponds to the theoretical prediction $\chi_{\epsilon}=1 / \epsilon$. 
appeared immediately that directed sandpile models, whose dynamics acts on a preferred direction, belong to a separate universality class [45]. On the contrary, early numerical simulations supported the belief that the Manna and BTW models belong to the same universality class $[46,47]$. A few years ago, larger numerical simulations [57] provided some evidence of two distinct universality classes. A better understanding of universality classes could be obtained using the framework discussed in this paper, studying the scaling with respect to the control parameters.

\section{On the role of conservation}

In the previous discussion, we emphasized the role of conservation in the dynamics of sandpile models. Using conservation laws, we found a subset of critical exponents that retain their mean-field values even in low dimensions. Conservation has an important effect on criticality as well, since the amount of dissipation plays the role of control parameter. Only by tuning this parameter to zero does the system become critical.

The role of conservation in SOC models has been the object of a long controversy. It was first claimed that conservation was a necessary condition for criticality in this class of model [21,22]. Results on dissipative sandpile models seemed to confirm this conclusion [50]. Later on, simulation of an earthquake model contradicted these results [59]. The model studied in Ref. [59] is a continuous height sandpile model in which energy is dropped uniformly over the entire lattice at an infinitesimal rate. This in practice corresponds to raising the heights of all the sites by the quantity needed for the higher site to become unstable. When a site $i$ is unstable $\left(z_{i}>z_{c}\right)$, the relaxation rules are

$$
\begin{gathered}
z_{i} \rightarrow 0, \\
z_{j} \rightarrow z_{j}+\alpha z_{i},
\end{gathered}
$$

where $j$ 's are the nearest neighbors of $i$. The dissipation parameter $\alpha[60]$ can be tuned: for $\alpha=1 /(2 d)$, the system is conservative. It has been observed in simulations that, in $d$ $=2$ for $\alpha>\alpha_{c}$, the system is critical. There is still not agreement on the precise value of $\alpha_{c}$, which was first estimated as $\alpha_{c} \simeq 0.05$ [59], and later found to be higher $\left(\alpha_{c} \simeq 0.18\right.$ ) [61], while it was claimed in Ref. [62] that $\alpha_{c}=0$.

The mean-field analysis of this model is not easy, because of the continuous number of levels a site can assume. Using an approximate analysis of the random neighbor model, it was claimed in Ref. [63] that $\alpha_{c} \simeq 0.22$, a value that was found to be in agreement with simulations. A complete analysis of the master equation later revealed that $\alpha_{c}=0.25$ (the conservative case), showing also the presence of very strong finite size correction [64]. From this analysis, it appears that the random neighbor model behaves like the BTW model. Criticality is only reached in the conservative case, in the limit of a zero driving rate. The situation in two dimensions is still controversial, and it is believed that the inhomogeneity created by the open-boundary conditions is responsible for the observed power law distributions.

The role of conservation for criticality still remains open in these models, while it is now agreed that in MF theory conservation is a necessary condition to achieve criticality in sandpile models. In Sec. V, we will discuss a model [37] that shows criticality without conservation even in MF theory. The price of achieving this result will be an additional driving rate.

\section{NONCONSERVATION AND CRITICALITY: THE FOREST-FIRE MODEL}

We have discussed that conservation in sandpiles is crucial to achieve criticality. The controversial issue of continuously driven models raises the question of the possibility that time scale separation alone can produce scale invariance in systems without conservation laws. In this context the forestfire model acquires a very important role, in that it is a nonconservative automaton displaying criticality.

As outlined in Sec. III, we can describe the model in the same language used for sandpile models. We identify burning sites with the active sites, since they interact with other sites independently of the driving fields. Furthermore, their density vanishes in the limit of small driving field $f$. In the same way, trees correspond to critical sites, and empty sites to stable sites. In this case the general three state description is exact. Using this language, we can emphasize differences and analogies between FF and sandpile models. While the main dynamical transitions are very similar, we can immediately recognize the effect of nonconservation. In the FF model energy in not stored, and critical sites are created by a second independent field: the tree growth probability $p$. Thus in the FF model we replace $h \rightarrow f$ and $u h \rightarrow p$. This introduces an independent field (or a time scale $p^{-1}$ ) related to the injection of critical sites in the system. Since energy is not accumulated, there is no need of an additional dissipation, so that in the FF model there is no parameter playing the role of $\epsilon$.

The FF model was originally introduced in the limit $f=0$ and $p \rightarrow 0$ by Bak, Chen, and Tang in Ref. [51]. The model was claimed to show SOC, but later Grassberger and Kantz [53] showed that in $d=2$ the model was critical in a trivial sense. The system shows a diverging characteristic length that is essentially the distance between straight fire fronts. This implies that the dynamics is governed by the average tree density over larger and larger regions. In higher dimensions, the possibility of a nontrivial behavior has not been ruled out, as recent work seems to suggest [54]. Drossel and Schwabl [52] introduced the ignition or lightning probability $f$. This field sustains fires, and the system flows in a stationary state which shows critical properties in the double limit $f \ll p \ll 1$. This version of the model has been the subject of several studies, both analytical $[12,27,65-67]$ and numerical [68-70].

Despite the various efforts, the two versions of the model were always studied as very different cases, almost two different models. For this reason, it is difficult to find in the literature a precise connection among the two different regimes. In this section we recover, within our framework, many results already present in the literature. By recasting these results in the language developed for sandpile automata, we provide a unified picture of both models. We discuss the FF model in terms of the response function singularities and we show that the SOC-FF and the deterministic FF correspond to the supercritical and subcritical regimes. 
In this way, we can understand several features of the FF model in terms of the same concepts developed for sandpile model.

The MF equations can be derived by the single site approximation to the master equation. Since the derivation proceeds as in Sec. IV, we do not repeat it here in detail, and we instead present some general considerations. Active sites become stable (fire $\rightarrow$ empty) with unitary rate, and critical sites become active if ignited by the lightning with probability $f$. The interaction term is then given by the fire spreading; an active site creates as many new active sites as the number of NN critical sites. To the first order in $\rho_{a}$, this term is proportional to $\rho_{a} \rho_{c}$ times the usual geometrical factor $g$ that takes into account the lattice coordination and other model dependent geometrical effects. The reaction rate equations then reads

$$
F_{a}=-\rho_{a}+f \rho_{c}+g \rho_{c} \rho_{a}+O\left(\rho_{a}^{2}\right) .
$$

This expression is very similar to the one obtained in the sandpile case, with the exception of the dissipative term that here is missing. The two models differ in the dynamical evolution of stable sites. In the FF model there is a term, due of the field $p$, corresponding to the transition rate from stable to critical sites, and there is no interaction between active and stable sites. We can then write the stationarity equations for the FF model as

$$
\begin{gathered}
\rho_{a}=f \rho_{c}+g \rho_{c} \rho_{a}, \\
\rho_{a}=p \rho_{s}, \\
\rho_{a}=1-\rho_{s}-\rho_{c},
\end{gathered}
$$

where we have neglected the second order term in $\rho_{a}$. As for the sandpile model, $g$ is an independent parameter of the model, and $f$ and $p$ represent the tunable external driving fields. The lowest order solutions in $f$ and $p$ to the above equations are

$$
\begin{gathered}
\rho_{a}=\frac{g-1}{g} p+\frac{1}{g} f+O\left(f^{2}, p^{2}\right), \\
\rho_{c}=\frac{1}{g}-\frac{1}{g} \frac{f}{p}+O(p, f), \\
\rho_{s}=\frac{g-1}{g}+\frac{1}{g} \frac{f}{p}+O(p, f) .
\end{gathered}
$$

These results were already obtained in Ref. [27], where a random neighbor version of the $\mathrm{FF}$ is analyzed. Their method and the present MF scheme are equivalent, and we will recover the same stationary densities. We compute the critical exponents by using the same lines adopted for sandpiles, and obtain some insight into the critical properties of the FF model. The density of active sites depends linearly upon $f$ and $p$, which are independent driving fields playing the same role as $h$ in sandpile automata. If we consider the density of active sites as the order parameter, it appears immediately that the critical point is reached if $f \rightarrow 0$ and $p$ $\rightarrow 0$ simultaneously. This double limit again corresponds to the locality breaking of the dynamical rules. In this case the order parameter is identically zero in the steady state, and the system develops long-range correlation properties. Also, for the FF model we can then distinguish among subcritical and supercritical regimes depending on the values of the driving fields.

\section{A. Subcritical regime}

The subcritical and critical regimes correspond to the limit in which we have a zero order parameter and therefore $f=0$ and $p=0$. This limit is, however, not completely defined because the density of critical and stable sites depend upon the ratio $f / p$. In order to study the critical behavior in this limit, we repeat the discussion inspired by the study of CA with adsorbing states that we already used for the sandpiles. Since when $f=0$ and $p=0$ the dynamics is frozen, we have to prepare the system in a stationary state in the limits $p \rightarrow 0$ and $f \rightarrow 0$, and then study the spreading of small perturbations. This is what is actually done in numerical simulations, where the fire evolution and the action of $f$ and $p$ act separately. In doing that, however, we prepare the system in one of the "natural" configurations, corresponding to the stationary state in the limit of infinitesimal driving. In this configuration the density of critical sites reaches a limit value $\rho_{c}=1 / g-g^{-1} f / p$, which depends, via the parameter $\theta$ $\equiv f / p$, on the way the limit has been performed.

In order to study the scaling behavior, we consider the limit $f \ll p \ll \theta \ll 1$, keeping $\theta$ constant. In this regime we can consider $f=p=0$ and $\rho_{a}=0$, and we can study the system to the leading order in $\theta$. By considering small deviations $\delta \rho_{\kappa}(t)$ from the stationary state, and retaining just first order terms in $\theta$, we find the linearized dynamical equation in diagonal form

$$
\frac{\partial}{\partial t} \delta \rho_{a}(t)=-\theta \delta \rho_{a}(t)
$$

Hence the relaxation behavior follows an exponential law in which the characteristic relaxation time is given by $t_{c}$ $\sim \theta^{-\Delta}$ with $\Delta=1$. This implicitly tells us that the system indeed reacts in avalanches. In fact, both driving time scales $p^{-1}$ and $f^{-1}$ are much larger in this regime than the characteristic spreading time of an avalanche $\theta^{-1}$, that therefore remains an isolated event connected in space and time, i.e., it does not overlap spatially with other avalanches or growth processes due to the driving field.

Along the lines we followed for sandpiles, we define the response function of the system $\chi_{f, \theta}\left(x-x^{\prime} ; t-t^{\prime}\right)$ that characterizes the way the system responds to an external perturbation. The response is now a function of $f$ and $\theta$. The total susceptibility $\chi_{f, \theta}$ is related to the derivative of the stationary density of critical sites, and the zero field susceptibility can be obtained as

$$
\chi_{\theta}=\lim _{f \rightarrow 0} \frac{\partial \rho_{a}(f)}{\partial f} .
$$

Since the density of active sites can be written as $\rho_{a}(f, \theta)$ $=f / g+(g-1) f /(g \theta)$, the singular part of the susceptibility diverges as 


$$
\chi_{\theta}=\theta^{-1}
$$

In Appendix A it is shown that the zero field susceptibility is related to the divergence of the average fire size as $\langle s\rangle$ $\sim \chi_{\theta}$. Hence the characteristic fire size diverges for $\theta \rightarrow 0$. This implies that the system is in a subcritical regime, and perturbations to the stationary state show a finite characteristic length for any $\theta>0$. Only in the limit $\theta \rightarrow 0$ does the system respond on all length scales to infinitesimal perturbations. We can define the standard scaling laws $\chi_{\theta}=\theta^{-\gamma}$ with $\gamma=1$, and $\xi \sim \theta^{-\nu}$ that characterize the divergence of the correlation length.

Next we consider the total response at a position $r$ given by $\chi_{\epsilon}(r)=\int \chi_{\epsilon}(r, t) d t$. We note that fire clusters are given by the connected clusters of critical sites, because in this regime fires are not overlapping. Since a tree can burn just once, the average response at a distance $r$ is given by the pair connectedness function that gives the probability that two sites at a distance $r$ are connected, i.e., belong to the same cluster. This function and thus the response function is supposed to behave as $r^{2-d} \Gamma(r / \xi)$ in MF theory [71]. In general, by integrating the local response function, we have

$$
\chi_{\theta}=\xi^{2}
$$

and, therefore, by comparing with Eq. (64), we obtain $\nu=$ $\frac{1}{2}$. It is worth remarking that in this case the above MF relations are not enforced by conservation laws, and anomalous exponents can appear in low dimensions.

To study the avalanche behavior, we introduce the probability $P(s, \theta)=s^{-\tau} \mathcal{G}\left(s / s_{c}(\theta)\right)$ that a fire involves $s$ sites, and we identify the usual set of critical exponents defined by the scaling laws $s_{c} \sim \theta^{-1 / \sigma}, s_{c} \sim \xi^{D}$, and $t_{c} \sim \xi^{z}$. Associated with these we have the scaling relations $D \sigma=1 / \nu, z \nu=\Delta$, and $\gamma \sigma=2-\tau$. We have shown previously that in this regime the class of "natural" configurations have a density of critical sites which depends on $\theta$; thus we consider the difference of densities with respect to the critical state

$$
\rho_{c}-\rho_{c}(\theta) \sim \theta^{\zeta}, \quad \zeta=1 .
$$

We can then find another scaling relation that links the avalanche exponents to $\zeta$, noting that the avalanche size distribution corresponds to the distribution of connected critical site clusters. In Appendix B we derive this scaling relation, which results to be

$$
\zeta=\frac{\tau-1}{\sigma}
$$

Collecting all the results obtained above, we have the complete set of MF exponents:

$$
\begin{gathered}
\gamma=1, \quad \nu=\frac{1}{2}, \\
\tau=3 / 2, \quad D=4, \quad \sigma=\frac{1}{2}, \quad z=2 .
\end{gathered}
$$

Also in this case, as previously shown by several authors [27], the MF values correspond to those of mean-field percolation. It is important to stress again that in the FF case, the absence of a conservation constraint implies that MF values for critical exponents are not valid in low-dimensional system. Anomalous scaling appears below the upper critical dimension, and the model shows nontrivial values of exponents [37].

\section{B. Supercritical regime}

Here we consider the scaling behavior in the region in which the order parameter is not zero. In order to remain in the critical region, we must have $\theta \ll 1$, but now we consider nonvanishing $f$ and $p$, with $f$ much smaller than $p$. This essentially corresponds to the FF model without ignition, that in this perspective can be considered as the supercritical regime close to the critical point. In this limit, we obtain immediately from the solution of Eq. (61) that the order parameter is positive, and scales as

$$
\rho_{a} \sim p^{\beta}
$$

with $\beta=1$. To calculate the relaxation properties we have to perform a linear stability analysis of the system around the stationary solutions (61), retaining only the lowest order terms in $p$. We consider small fluctuations $\delta \rho_{\kappa}(t)$, and the eigenvalues of the diagonal form of the dynamical evolutions are

$$
\Lambda_{ \pm}=-(g / 2) p \pm i(g p)^{1 / 2}
$$

The negative real part identifies the characteristic relaxation time that scales as $t_{c} \sim p^{-1}$. Together with the exponential relaxation, the system shows oscillations with period $T$ $\sim p^{-1 / 2}$, related to the imaginary part of the eigenvalues. This MF behavior was already discussed in Ref. [53].

In the supercritical state the time scale of a perturbation is comparable to the driving scale, both being of the order of $p^{-1}$. Thus active sites do not spread just on connected clusters of critical sites. In other words, the critical site configuration is not frozen during the perturbation, and the time evolution connects several clusters of critical sites because connecting sites might appear during the time evolution. Also in this case, however, the susceptibility is given by the total response to a localized fluctuation,

$$
\chi_{p} \sim \int \delta \rho_{a}(t) d t \sim p^{-1}
$$

Since the response of the system is due to the connectivity properties, we have still the usual MF relation $\chi_{p} \sim \xi^{2}$, which implies that $\xi \sim p^{-\nu^{\prime}}$ with $\nu^{\prime}=\frac{1}{2}$. Another way to see this result is to think that the fluctuation spreads as waves of active sites. Since the propagation velocity is finite, the correlation length is proportional to the wave period $T$. This simple MF picture does not work in low dimensions [54].

\section{DISCUSSION AND OPEN QUESTIONS}

\section{A. Relations with branching processes}

A clear mean-field description of the avalanches in SOC models has been obtained through mapping to branching processes [29-32]. A branching process [72] is defined by a number of active sites that can either die or generate $n$ new sites with certain probabilities. The simpler example is the case $n=2$ : a site dies with probability $1-q$ or generates two 
new sites with probability $q$. The process usually starts with a single active site and continues until no more active sites are present. Depending on the value of $q$, the branching process will die after a finite number of steps or continue forever. There is a critical value $q=q_{c}$ that separates the two regimes $\left(q_{c}=\frac{1}{2}\right.$ for $\left.n=2\right)$. For $q<q_{c}$, the size distribution of the branching process is a power law

$$
P(s) \sim s^{-3 / 2} f\left(s / s_{0}\right),
$$

where the cutoff $s_{0}$ diverges for $q=q_{c}$.

It was shown in Ref. [31] that the Manna model can be exactly mapped into a branching process with a time dependent parameter $q(t)$, depending on the density of critical sites $\left(\rho_{c}\right)$ and on the dissipation [32]. A critical branching process was obtained as a stationary state in the limit of slow driving and conservation [31].

Branching processes can be considered as a general framework to describe avalanches in mean-field theory. In general terms, we can describe an avalanche by an evolving front that can either propagate or stop. In the mean-field description, the elements of the fronts do not interact and evolve independently. Thus the avalanche can be described as a branching process with an effective parameter $q$ that depends on the detail of the model under study.

In our formalism, a branching process is associated with the propagation of active sites in the subcritical regime. In the stationary state for $h=0$, an active site generates $k$ $=1, \ldots, g$ new active sites with probabilities

$$
q_{k}=(1-\epsilon)\left(\begin{array}{l}
g \\
k
\end{array}\right) \rho_{c}^{k}\left(1-\rho_{c}\right)^{g-k},
$$

while no active sites are generated with probability

$$
q_{0}=\epsilon+(1-\epsilon)\left(1-\rho_{c}\right)^{g} .
$$

In this case, the control parameter for the branching process is given by $\tilde{q}=\sum_{k} k q_{k}$, with a critical value $\tilde{q}_{c}=1$. In the stationary state, we find $\rho_{c}=1 / g$ and hence $\tilde{q}=1-\epsilon$. The critical branching process corresponds, therefore, to the limit $\epsilon \rightarrow 0$. A similar analysis can be done for the FF model.

\section{B. Locality breaking}

We have seen that criticality in stochastic SOC systems is achieved only in the limit of infinitesimal driving corresponding to the locality breaking of the dynamical rules. The nonlocality is evident if we consider the zero driving limit that is naturally implemented in computer simulations using two different time scales, one for the avalanche evolution and one for the driving. With this infinite time scale separation, the evolution of each site depends on the entire system.

For a more concrete physical explanation of how the locality breaking generates long-range interactions in the system, let us consider the case of a vanishing driving rate, corresponding to a small density of active sites. Because of the infinitesimal driving, each region, devoid of active particle, is virtually frozen until an active site is generated. The activity spreads, and in general alters the configuration before it moves away or disappears. The active sites leave a trace of their dynamical history in the frozen configurations of critical and stable sites they produced. If new active sites are created in the same region at some later times, they can "feel" the effect of the active sites present earlier in the region. This is basically a memory effect, which creates a long-range interaction in time and space among diffusing active sites. The range of this interaction depends on the characteristic time scale of the driving, because the fluctuations induced by the driving destroy the memory effect.

Close to the infinite time scale separation, the characteristic driving time scale is diverging, and the range of the nonlocal interaction extends to the entire system. A local interaction is recovered, however, if we introduce a size cutoff in the wandering region of active particles. This is the case of dissipative sandpile in which after a finite number of steps the active sites disappear [73]. Over this characteristic, size active particles do not interact, and to obtain a longrange nonlocal interaction the dissipation should go to zero. The same discussion applies to the FF model, due to the finite range of connected critical sites obtained by tuning the ratio of $f$ and $p$.

\section{Conclusions}

In this paper, we have presented a unified mean-field theory for stochastic SOC models. We have treated these models in analogy with other nonequilibrium cellular automata, using a single site approximation to the master equation. With the present approach, we are able to identify the order parameter and the control parameters of the models and to emphasize similarities and differences between SOC and other nonequilibrium system. In particular, the language of cellular automata with absorbing states can be employed to describe SOC models. For finite driving rates, we find a supercritical regime characterized by a finite fraction of active sites. In the limit of infinitesimal driving, the system is subcritical and displays an avalanche response. Criticality arises from a double limit: the driving rate and the dissipation (in the sandpile model) or the two driving rates (in the FF model) should have vanishing values. This limit corresponds to the onset of nonlocal dynamical rules, which are responsible for the critical behavior characteristic of SOC.

From this perspective, SOC models appear to be nonequilibrium systems with steady states, reaching criticality by the fine tuning of control parameters. While this statement is technically correct, we note that SOC systems are quite peculiar, since the fine tuning can only be achieved by limit procedure. This is in contrast with ordinary critical phenomena, where the control parameter can be directly tuned to its critical value. In this sense, SOC systems are less sensitive to fine tuning [10]. Moreover, the driving rate can in general be small in many natural phenomena, and this could make the SOC framework relevant.

\section{ACKNOWLEDGMENTS}

We thank G. Caldarelli, A. Chessa, K. B. Lauritsen, V. Loreto, E. Marinari, A. Maritan, M.A. Mữoz, A. Omar Garcia, and L. Pietronero. We are grateful to R. Dickman for valuable comments and suggestions. A. V. is indebted to $\mathrm{J}$. M. J. van Leeuwen for interesting discussions. We thank the Instituut-Lorentz in Leiden, where part of this work was 
completed. The Center for Polymer Studies was supported by the NSF.

\section{APPENDIX A: RESPONSE FUNCTION PROPERTIES}

For small perturbations around the stationary state, the spontaneous microscopic dynamics can be represented by introducing the response function. We first consider the sandpile case. If we apply a time dependent perturbation $h(x, t)$ to the stationary state, the density of active sites changes as

$$
\begin{aligned}
\Delta \rho_{a}(x, t)= & \iint \chi_{h, \epsilon}\left(x-x^{\prime} ; t-t^{\prime}\right) \Delta h\left(x^{\prime}, t^{\prime}\right) d^{d} x^{\prime} d t^{\prime} \\
& +O\left((\Delta h)^{2}\right)
\end{aligned}
$$

where $\chi_{h, \epsilon}\left(x-x^{\prime} ; t-t^{\prime}\right)$ is the response or generalized susceptibility function. Here we assume a stationary and homogeneous system, i.e., the two point averages depend just on the time or space displacement. The above expression is valid in the linear regime, only for small variations of the perturbing field. We next derive some simple properties of the response function for systems whose dynamics is characterized by avalanches. We first consider an impulsive disturbance $\Delta h\left(x^{\prime}, t^{\prime}\right)=\delta(t) \delta^{d}(x)$. This is a very small perturbation with respect to the total energy input $J=\int h(x) d^{d} x$. In practice, it corresponds to the addiction of an energy grain on top of the stationary average driving field. Inserting this perturbation into $\Delta \rho_{a}(x, t)$ yields

$$
\Delta \rho_{a}(x, t)=\chi_{h, \epsilon}(x ; t) .
$$

We then define the total susceptibility of the system,

$$
\chi_{h, \epsilon}=\int d t \int \chi_{h, \epsilon}(x ; t) d^{d} x,
$$

which quantifies the total response of the system to an impulsive disturbance. The total number of active sites due to the perturbation is

$$
N_{a}=\int d t \int \Delta \rho_{a}(x, t) d^{d} x=\chi_{h, \epsilon} .
$$

In the absence of an external field $h \rightarrow 0$, the only active sites present in the system are due to the $\delta$ perturbation. That is, all the active sites are casually connected in space and time, thus forming an avalanche whose average size is $\langle s\rangle=N_{a}$. This is precisely stated by the expression

$$
\chi_{\epsilon} \equiv \lim _{h \rightarrow 0} \chi_{h, \epsilon}=\langle s\rangle,
$$

which defines a relation between the average avalanche size and the zero field susceptibility. As we have seen in the previous sections, the above expression is at the basis of several scaling relations, and it explains, together with conservation, the diffusive behavior of the average activity.

Another way to look at a stationary perturbation or equivalently to the variation of the stationary averages is the following. We consider a different perturbation

$$
\Delta h\left(x^{\prime}, t^{\prime}\right)=\Delta h \quad \text { for } t^{\prime}<t,
$$

corresponding to a uniform driving in space and time. By changing the variables of integration to $t^{\prime \prime}=t-t^{\prime}$ and $x^{\prime \prime}$ $=x-x^{\prime}$, we obtain

$$
\Delta \rho_{a}=\Delta h \int_{V} \int_{0}^{\infty} \chi_{h, \epsilon}\left(x^{\prime \prime} ; t^{\prime \prime}\right) d^{d} x^{\prime \prime} d t^{\prime \prime} .
$$

Hence the density fluctuations are time and space independent, as it must be in the new stationary state with $h \rightarrow h$ $+\Delta h$. Performing the double integral in the right term, we obtain the total susceptibility. Therefore,

$$
\Delta \rho_{a}=\Delta h \chi_{h, \epsilon},
$$

from which we obtain that in the stationary state and for infinitesimal perturbations,

$$
\chi_{h, \epsilon}=\lim _{\Delta h \rightarrow 0} \frac{\Delta \rho_{a}}{\Delta h}=\frac{\partial \rho_{a}(h)}{\partial h} .
$$

Notice that from this equation we are able to provide a relation between the total response function and the divergence of avalanche size

$$
\langle s\rangle=\chi_{\epsilon}=\lim _{h \rightarrow 0} \frac{\partial \rho_{a}(h)}{\partial h} .
$$

Equation (A10) states that the zero field susceptibility in the stationary state and the average avalanche size have the same singular behavior in the thermodynamic limit.

We next consider the forest-fire model. In this case we have the two driving fields $f$ and $p$, and the response function depends upon them. The interesting subcritical regime is the one in which we take the limit $f \rightarrow 0$ and $p \rightarrow 0$ with $\theta$ $=f / p \ll 1$. We study the response of the system for small perturbation $\Delta f$ and a fixed value of $\theta$. The general expression that characterizes the response of the system is given by

$$
\begin{aligned}
\Delta \rho_{a}(x, t)= & \iint \chi_{f, \theta}\left(x-x^{\prime} ; t-t^{\prime}\right) \Delta f\left(x^{\prime}, t^{\prime}\right) d^{d} x^{\prime} d t^{\prime} \\
& +O\left((\Delta f)^{2}\right) .
\end{aligned}
$$

As for the the sandpile case, we can apply a $\delta$ perturbation. It follows that, simply rewriting what we derived in the sandpile case, we obtain

$$
\chi_{\theta} \equiv \lim _{f \rightarrow 0} \chi_{f, \theta}=\langle s\rangle,
$$

where $\langle s\rangle$ in this case is the average size of fire events. In the same way we can consider a stationary perturbation $\Delta f\left(x^{\prime}, t^{\prime}\right)=\Delta f$ for $t^{\prime}<t$, and, by repeating the above arguments, we recover

$$
\chi_{f, \theta}=\frac{\partial \rho_{a}(f)}{\partial f},
$$

from which follows that the divergence of the average size of fires is related to the zero field susceptibility in the usual way. 


\section{APPENDIX B: SCALING RELATIONS}

Here we obtain two scaling relations whose derivation is straightforward but rather lengthy.

\section{Sandpile model}

Let us consider the flow decays of activity in the subcritical regime. We define $\rho_{a}(s, t)$, the space integrated response of an avalanche of $s$ sites. If we assume scaling behavior, we have that

$$
\rho_{a}(s, t) \simeq s^{q} w\left(t / t_{s}\right),
$$

where $t_{s}$ is the upper characteristic time of an avalanche of $s$ sites and scales as $t_{s} \sim s^{z / D}$. By imposing the condition that $\int \rho_{a}(s, t) d t=s$, we obtain $q=1-z / D$. We have also that $w(0)=w(1)=0$, and that $\rho_{a}(s, t)$ is independent of $s$ for small $t$. This implies that $w(x) \rightarrow x^{-1+D / z}$ as $x \rightarrow 0$. The total response function is the average of the various possible avalanche response

$$
\rho_{a}(t)=\int \rho_{a}(s, t) P(s) d s,
$$

which, after the proper substitution by means of scaling relations, gives the expression

$$
\rho_{a}(t) \sim \exp \left[-\left(t / t_{c}\right)^{(\tau-1) / \nu \sigma z}\right] .
$$

In the MF picture, the above relation is consistent with the results obtained from the dynamical equations only if

$$
\frac{(\tau-1)}{\nu \sigma z}=1
$$

thus recovering the relations used in Sec. IV. An analogous result was already obtained in the paper by Tang and Bak [35]. Again, we stress that this is not a general scaling relation, but an exponent equality valid just in MF theory.

\section{Forest-fire model}

The density of critical sites in the stationary configuration approaches the critical value for $\theta \rightarrow 0$ as a power law

$$
\Delta \rho_{c} \equiv \rho_{c}(\theta=0)-\rho_{c}(\theta) \sim \theta^{\zeta} .
$$

In the subcritical regime, we have a complete time scale separation. Therefore, each spreading of activity involves just clusters of connected critical sites. This is because the tree growth time scale $p^{-1}$ is much longer than the activity time scale, thus preventing new critical sites from changing the connectivity properties of the configuration. In this condition, the probability $P(s, \theta)$ to have an avalanche of size $s$ scales as the distribution $n(s)$ of connected clusters with $s$ critical sites times the size of the cluster $s$. This factor takes into account the probability that the ignition process starts on any site of a cluster of size $s$. On the other hand, the density of critical sites, leaving apart normalization factors, is given by $\rho_{c} \sim \int s n(s) d s$; that is, given by the integral of the avalanche distribution. We can therefore write

$$
\Delta \rho_{c} \sim \int s^{-\tau}\left[1-\mathcal{G}\left(s / s_{c}(\theta)\right)\right] d s,
$$

where we used the explicit form of the avalanche distribution. Noticing that $\mathcal{G}\left(s / s_{c}(\theta)\right) \simeq 0$ for $s>s_{c}$, we obtain that the main contribution to the above integral is given by

$$
\Delta \rho_{c} \sim \int_{s_{c}}^{\infty} s^{-\tau} d s,
$$

or, as a result of the integration,

$$
\Delta \rho_{c} \sim s_{c}^{1-\tau} .
$$

By substituting $s_{c} \sim \theta^{-1 / \sigma}$ in the above expression and requiring the scaling consistency with Eq. (B5), we finally obtain the scaling relation

$$
\zeta=\frac{\tau-1}{\sigma}
$$

[1] P. Bak, C. Tang, and K. Wiesenfeld, Phys. Rev. Lett. 59, 381 (1987); Phys. Rev. A 38, 364 (1988).

[2] G. Durin, G. Bertotti, and A. Magni, Fractals 3, 351 (1995); D. Spasojević, S. Bukvić, S. Milosević, and H. E. Stanley, Phys. Rev. E 54, 2531 (1996), and reference therein.

[3] S. Field, J. Witt, F. Nori, and X. Ling, Phys. Rev. Lett. 74, 1206 (1995).

[4] M. P. Lilly, P. T. Finley, and R. B. Hallock, Phys. Rev. Lett. 71, 4186 (1993); for a review, see M. Sahimi, Rev. Mod. Phys. 65, 1393 (1993)

[5] A. Petri, G. Paparo, A. Vespignani, A. Alippi, and M. Costantini, Phys. Rev. Lett. 73, 3423 (1994); S. Zapperi, A. Vespignani, and H. E. Stanley, Nature (London) 388, 658 (1997).

[6] G. Gutenberg and C. F. Richter, Ann. Geophys. (C.N.R.S.) 9, 1 (1956).

[7] B. Suki, A.-L. Barabasi, Z. Hantos, F. Petak, and H. E. Stanley, Nature (London) 368, 615 (1994).

[8] H. M. Jaeger, C. H.- Liu, and S. R. Nagel, Phys. Rev. Lett. 62, 40 (1989).

[9] V. Frette, K. Christensen, A.M. Malthe-Sørenssen, J. Feder, T.
Jøssang, and P. Meakin, Nature (London) 397, 49 (1996).

[10] G. Grinstein, in Scale Invariance, Interfaces and NonEquilibrium Dynamics, Vol. 344 of NATO Advanced Study Institute, Series B: Physics, edited by A. McKane et al. (Plenum, New York, 1995).

[11] D. Sornette, A. Johansen, and I. Dornic, J. Phys. I 5, 325 (1995).

[12] A. Vespignani, S. Zapperi, and V. Loreto, Phys. Rev. Lett. 77, 4560 (1996); J. Stat. Phys. 88, 47 (1997).

[13] A. Vespignani and S. Zapperi, Phys. Rev. Lett. 78, 4793 (1997).

[14] B. Schmittmann and R. K. Zia, in Phase Transitions and Critical Phenomena, edited by C. Domb and J. L. Lebowitz (Academic, London, 1995), Vol. 17.

[15] D. Sornette, J. Phys. I 2, 2065 (1992).

[16] L. Gil and D. Sornette, Phys. Rev. Lett. 76, 3991 (1996).

[17] D. Dhar, Phys. Rev. Lett. 64, 1613 (1990).

[18] D. Dhar and S. N. Majumdar, J. Phys. A 23, 4333 (1990); S. N. Majumdar and D. Dhar, ibid. 24, L357 (1991); Physica A 185, 129 (1992). 
[19] V. B. Priezzhev, J. Stat. Phys. 74, 955 (1994); E. V. Ivashkevich, J. Phys. A 27, 3643 (1994); E. V. Ivashkevich, D. V. Ktitarev, and V. B. Priezzhev, Physica A 209, 347 (1994).

[20] Y. C. Zhang, Phys. Rev. Lett. 63, 470 (1989); L. Pietronero, P. Tartaglia, and Y. C. Zhang, Physica A 173, 129 (1991).

[21] T. Hwa and M. Kardar, Phys. Rev. Lett. 62, 1813 (1989); Phys. Rev. A 45, 7002 (1992).

[22] G. Grinstein, D.-H. Lee, and S. Sachdev, Phys. Rev. Lett. 64, 1927 (1990).

[23] A. Díaz-Guilera, Phys. Rev. A 45, 8551 (1992); Europhys. Lett. 26, 177 (1994).

[24] L. Pietronero, A. Vespignani, and S. Zapperi, Phys. Rev. Lett. 72, 1690 (1994); A. Vespignani, S. Zapperi, and L. Pietronero, Phys. Rev. E 51, 1711 (1995); E. V. Ivashkevich, Phys. Rev. Lett. 76, 3368 (1996); J. Hasty and K. Wiesenfeld, J. Stat. Phys. 86, 1179 (1997).

[25] S. A. Janowsky and C. A. Laberge, J. Phys. A 26, L973 (1993).

[26] H. Flyvbjerg, K. Sneppen, and P. Bak, Phys. Rev. Lett. 71, 4087 (1993); J. de Boer, B. Derrida, H. Flyvbjerg, A. D. Jackson, and T. Wettig, ibid. 73, 906 (1994).

[27] K. Christensen, H. Flyvbjerg, and Z. Olami, Phys. Rev. Lett. 71, 2737 (1993).

[28] K. Christensen and Z. Olami, Phys. Rev. E 48, 3361 (1993).

[29] P. Alstrøm, Phys. Rev. A 38, 4905 (1988).

[30] R. García-Pelayo, Phys. Rev. E 49, 4903 (1994).

[31] S. Zapperi, K. B. Lauritsen, and H. E. Stanley, Phys. Rev. Lett. 75, 4071 (1995).

[32] K. B. Lauritsen, S. Zapperi, and H. E. Stanley, Phys. Rev. E 54, 2483 (1996).

[33] M. Vergeles, A. Maritan, and J. R. Banavar, Phys. Rev. E 55, 1998 (1997); G. Caldarelli (unpublished).

[34] M. Katori and H. Kobayashi, Physica A 229, 461 (1996).

[35] C. Tang and P. Bak, Phys. Rev. Lett. 60, 2347 (1988); J. Stat. Phys. 51, 797 (1988).

[36] A. L. Stella, C. Tebaldi, and G. Caldarelli, Phys. Rev. E 52, 72 (1995); G. Caldarelli, A. Maritan, and G. Vendruscolo, Europhys. Lett. 35, 481 (1996).

[37] For a review, see S. Clar, B. Drossel, and F. Schwabl, J. Phys.: Condens. Matter 8, 6803 (1996).

[38] M. Paczuski, S. Maslov, and P. Bak, Phys. Rev. E 53, 414 (1996).

[39] P. Grassberger and Y. C. Zhang, Physica A 224, 169 (1996); D. Sornette and I. Dornic, Phys. Rev. E 54, 3334 (1996).

[40] P. Grassberger and A. de la Torre, Ann. Phys. (N.Y.) 122, 373 (1979)

[41] R. Dickman, Phys. Rev. A 34, 4246 (1986); 38, 2588 (1988); J. Stat. Phys. 55, 997 (1989); T. Tomé, Physica A 212, 99 (1994).

[42] J. F. Mendes, R. Dickman, M. Henkel, and M. Ceu Marques, J. Phys. A 27, 3019 (1994); M. A. Mũ̃oz, G. Grinstein, R. Dickman, and R. Livi, Phys. Rev. Lett. 76, 451 (1996).

[43] D. Wilkinson and J. F. Willemsen, J. Phys. A 16, 3365 (1983).

[44] P. Bak and K. Sneppen, Phys. Rev. Lett. 71, 4083 (1993).

[45] L. P. Kadanoff, S. R. Nagel, L. Wu, and S. Zhu, Phys. Rev. A 39, 6524 (1989).

[46] P. Grassberger and S. S. Manna, J. Phys. (France) 51, 1077 (1990).

[47] S. S. Manna, J. Stat. Phys. 59, 509 (1990); Physica A 179, 249 (1991)

[48] S. Lübeck and K. D. Usadel, Phys. Rev. E 55, 4095 (1997); cond-mat/9708055 (1997); S. Lübeck, Phys. Rev. E 56, 1590 (1997).

[49] S. S. Manna, J. Phys. A 24, L363 (1991).

[50] S. S. Manna, L. B. Kiss, and J. Kertész, J. Stat. Phys. 61, 923 (1990).

[51] P. Bak, K. Chen, and C. Tang, Phys. Lett. A 147, 297 (1990).

[52] B. Drossel and F. Schwabl, Phys. Rev. Lett. 69, 1629 (1992).

[53] P. Grassberger and H. Kantz, J. Stat. Phys. 63, 685 (1991).

[54] H.-M. Broker and P. Grassberger, Phys. Rev. E 59, R4918 (1997).

[55] $J_{\text {in }}=h L^{d}$ is the average energy added considering just first order contributions in $h$ and $\rho_{a}$. In fact, energy is not usually added on top of already active sites. This makes the energy addiction $J_{\text {in }}=h\left(1-\rho_{a}\right) L^{d}$. Also, the energy balance in the stationary state is changed accordingly, thus obtaining $h(1$ $\left.-\rho_{a}\right) L^{d}=\epsilon \rho_{a} L^{d}$. The latter immediately gives the stationary density of active sites as $\rho_{a}=h /(h+\epsilon)$. For $\epsilon \gg h$, we recover the first order result presented in the text. However, higher order corrections can be considered, obtaining consistency relations useful for a higher order treatment of the MF equations.

[56] A. Vespignani and S. Zapperi (unpublished).

[57] A. Ben-Hur and O. Biham, Phys. Rev. E 53, R1317 (1996).

[58] A. Chessa, E. Marinari, A. Vespignani, and S. Zapperi (unpublished).

[59] Z. Olami, H. J. S. Feder, and K. Christensen, Phys. Rev. Lett. 68, 1244 (1992).

[60] In our notation, $\alpha \equiv(1-\epsilon) /(2 d)$.

[61] P. Grassberger, Phys. Rev. E 49, 2436 (1994).

[62] A. A. Middleton and C. Tang, Phys. Rev. Lett. 74, 742 (1995).

[63] S. Lise and H. J. Jensen, Phys. Rev. Lett. 76, 2326 (1996).

[64] M. L. Chabanol and V. Hakim, Phys. Rev. E 56, R2343 (1997); P. Grassberger and H.-M. Broker, Phys. Rev. E 56, 3944 (1997).

[65] B. Drossel, S. Clar, and F. Schwabl, Phys. Rev. Lett. 71, 3739 (1993).

[66] V. Loreto, L. Pietronero, A. Vespignani, and S. Zapperi, Phys. Rev. Lett. 75, 465 (1995); V. Loreto, A. Vespignani, and S. Zapperi, J. Phys. A 29, 2981 (1996).

[67] H. Patzlaff and S. Trimper, Phys. Lett. A 189, 187 (1994).

[68] S. Clar, B. Drossel, and F. Schwabl, Phys. Rev. E 50, 1009 (1994).

[69] C. L. Henley, Phys. Rev. Lett. 71, 2741 (1993).

[70] P. Grassberger, J. Phys. A 26, 2081 (1993).

[71] The pair connectedness function is given by the percolation properties of tree sites in the presence of a characteristic length defined by the dynamical process. The general form of the pair connectedness is $r^{2-d-\eta_{\text {occ }}} \Gamma(r / \xi)$. In the MF description, where no anomalous dimension appears, $\eta_{\text {occ }}=0$. See J. W. Essam, in Phase Transitions and Critical Phenomena, edited by C. Domb and M. S. Green (Academic, London, 1972), Vol. 2 .

[72] T. E. Harris, The Theory of Branching Processes (Dover, New York, 1989).

[73] It is easy to recognize that, in a dissipative model, each active particle can wander only a finite number of steps. Each toppling process loses on average an amount $\epsilon$ of energy. The energy unit needed to sustain the activity (the wandering of the active site) is therefore dissipated after $\epsilon^{-1}$ toppling events (wandering steps). This indeed recovers the result obtained for the average avalanche size in our MF approach. 